\title{
Production of Highly Monolayer Enriched Dispersions of Liquid-Exfoliated Nanosheets by Liquid Cascade Centrifugation
}

Claudia Backes, ${ }^{1,2}$ Beata M. Szydłowska, ${ }^{1,2}$ Andrew Harvey, ${ }^{1,2}$ Shengjun Yuan, ${ }^{3}$ Victor VegaMayoral, ${ }^{4,5}$ Ben R. Davies, ${ }^{1,2}$ Pei-liang Zhao, ${ }^{6}$ Damien Hanlon,,${ }^{1,2}$ Elton J. G. Santos, ${ }^{7}$ Mikhail I. Katsnelson, ${ }^{3}$ Werner J Blau, ${ }^{1,2}$ Christoph Gadermaier, ${ }^{4,5}$ and Jonathan N. Coleman ${ }^{1,2 *}$ ${ }^{1}$ CRANN \& AMBER, Trinity College Dublin, Dublin 2, Ireland

${ }^{2}$ School of Physics, Trinity College Dublin, Dublin 2, Ireland

${ }^{3}$ Institute for Molecules and Materials, Radboud University of Nijmegen, Heijendaalseweg 135, 6525AJ Nijmegen, the Netherlands

${ }^{4}$ Department for Complex Matter, Jozef Stefan Institute, Jamova 39, 1000 Ljubljana, Slovenia ${ }^{5}$ Jozef Stefan International Postgraduate School, Jamova 39, 1000 Ljubljana, Slovenia ${ }^{6}$ Department of Applied Physics, Zernike Institute for Advanced Materials, University of Groningen, Nijenborgh 4, NL-9747AG Groningen, The Netherlands

${ }^{7}$ School of Mathematics and Physics and School of Chemistry and Chemical Engineering, Queen's University Belfast, Belfast, BT95AL, United Kingdom

*colemaj@tcd.ie

ABSTRACT: While liquid exfoliation is a powerful technique to produce defect-free nanosheets in large quantities, its usefulness is limited by broad nanosheet thickness distributions and low monolayer contents. Here we demonstrate liquid processing techniques, based on iterative centrifugation cascades, which can be designed to achieve either highly efficient nanosheet size-selection and/or monolayer enrichment. The resultant size-selected dispersions were used to establish quantitative metrics to determine monolayer volume fraction, as well as mean nanosheet size and thickness, from standard spectroscopic measurements. Such metrics allowed us to design and optimize centrifugation cascades to enrich liquid exfoliated $\mathrm{WS}_{2}$ dispersions up to monolayer contents of $75 \%$. Monolayer-rich dispersions show relatively bright photoluminescence with narrow linewidths $(<35 \mathrm{meV})$ indicating the high quality of the nanosheets. The enriched dispersions display extinction spectra with distinct features, which also allow the direct estimation of monolayer contents.

Keywords: exfoliation, monolayer, size-selection, luminescence 
Liquid phase exfoliation is a versatile technique for producing liquid suspensions containing large quantities of 2-dimensional (2D) nanosheets. ${ }^{1-7}$ This method involves the sonication $^{2,4}$ or shearing 8,9 of layered materials in liquids, resulting in the production of generally defect-free nanosheets. If the liquid is appropriately chosen (e.g. suitable solvents ${ }^{5,6}$, 10,11 or surfactant ${ }^{3,12-15}$ or polymeric solutions ${ }^{16-19}$ ) the nanosheets will be stabilized against reaggregation. ${ }^{20}$ This method has been used to exfoliate a broad range of $2 \mathrm{D}$ materials including graphene, ${ }^{2,6,9,12,17,21} \mathrm{BN},{ }^{5}$ transition metal dichalcogenides such as $\mathrm{MoS}_{2}$ and $\mathrm{WSe}_{2},{ }^{4,8,22,23}$ as well as $\mathrm{MoO}_{3},{ }^{24,}{ }^{25} \mathrm{GaS},{ }^{26}$ black phosphorous ${ }^{27-31}$ and MXenes. ${ }^{32}$ The liquid suspensions produced in this way are extremely useful, as they can be easily processed into a range of structures including films, coatings and composites. ${ }^{5,23,33-35}$ Such materials have demonstrated superlative performance in a number of applications including reinforced composites, ${ }^{5,18,36,37}$ battery electrodes ${ }^{38,39}$ and fiber lasers. ${ }^{40-42}$

However, liquid phase exfoliation tends to give nanosheets with very broad lateral size (length, L) and thickness (expressed as number of monolayers per nanosheet, $\mathrm{N}$ ) distributions, ${ }^{15,43}$ with ranges of $50 \mathrm{~nm} \leq \mathrm{L} \leq 500 \mathrm{~nm}$ and $1 \leq \mathrm{N} \leq 10$ not unusual for $\mathrm{MoS}_{2} \cdot{ }^{15}$ Such distributions are problematic as many applications require controlled nanosheet sizes: small nanosheets are ideal for catalysis ${ }^{44}$ while large ones are needed for mechanical reinforcement. ${ }^{37}$ These broad distributions also mean the monolayer content is always low, generally $<10 \%$. This is a serious problem for a number of applications. For example, the use of 2D materials in printed optoelectronic devices cannot be considered unless inks containing very high monolayer contents are available. ${ }^{45}$ In the longer term, nanosheet printed electronics will require monodisperse suspensions containing only $\mathrm{N}$-layers where $\mathrm{N}$ is defined by the application.

Such capabilities are a long way away. While some progress has been made toward selection of nanosheets by size, ${ }^{15,43}$ the available processes are generally inefficient, yielding very small quantities of nanosheets. In addition, monolayer enrichment is much more challenging, not least because the measurement of monolayer content generally involves statistical microscopy which is very time consuming. While density gradient ultracentrifugation has been used to separate TMD nanosheets by thickness, ${ }^{46}$ this procedure is complex, gives low yields (concentrations $<0.005 \mathrm{~g} / \mathrm{L}$ ) and is limited to polymer-stabilized dispersions. What is needed is a scalable, universally applicable, high-yield technique to either size-select nanosheets or produce highly monolayer-enriched dispersions. Any such process will involve optimization, which is limited by the tedious microscopic characterization 
required. Hence, to enable this, it will be necessary to develop fast techniques to measure nanosheet size, thickness and monolayer content. Within this manuscript we address these points.

Inspired by gas-separation centrifugation cascades, we have developed an efficient centrifugation-based method, which allows nanosheet dispersions to be both separated by size and enriched in monolayers in a controlled way. By studying the optical properties of $\mathrm{WS}_{2}$ dispersions as a function of nanosheet size, thickness and monolayer content, we identified spectral properties which scale with monolayer content as well as mean nanosheet length and thickness yielding quantitative metrics for these properties. The resultant ability to measure $\langle L\rangle,\langle N\rangle$ and monolayer content, allows us to design secondary cascades to further enhance the monolayer content, reaching values as high as $75 \%$. Such monolayer-rich dispersions display easily observable photoluminescence (PL) and optical properties that distinct from normally observed for $\mathrm{WS}_{2}$ ensembles.

\section{Results}

\section{Size selection of $\mathrm{WS}_{2}$ nanosheets}

As liquid-exfoliated nanosheets tend to be polydisperse in both size and thickness, size selection is almost always required. A number of size-selection techniques exist, mostly based on centrifugation. ${ }^{3,15,43,46,47}$ However, these techniques tend to be inefficient, yielding small masses of size-selected nanosheets. To address this, we have developed a new centrifugationbased technique which we denote liquid cascade centrifugation (LCC). We start with a dispersion of liquid-exfoliated nanosheets, obtained by sonication of $\mathrm{WS}_{2}$ powder in aqueous surfactant solution (see methods) where any unexfoliated crystallites had been removed by low speed centrifugation (here $1.5 \mathrm{krpm}$ in our centrifuge). This "stock" dispersion contains nanosheets with a broad distribution of sizes and thickness and a small but non-trivial population of monolayers with varying lateral sizes. The stock is then centrifuged at a higher speed (here $2 \mathrm{krpm}$ ) and the sediment collected. This sediment contains nanosheets from the larger end of the size distribution and, as they were collected between centrifugation rates of 1.5 and $2 \mathrm{krpm}$, we refer to this sample as " $1.5-2 \mathrm{krpm}$ ". Critical to LCC, this sediment can be redispersed ${ }^{48}$ completely by mild sonication in $\mathrm{H}_{2} \mathrm{O}-\mathrm{SC}$ (at $\mathrm{SC}$ concentrations as low as 0.1 $\mathrm{g} / \mathrm{L})$ to give dispersions with virtually any chosen concentration. The supernatant produced during the $2 \mathrm{krpm}$ centrifugation contains all but the largest nanosheets. It can be centrifuged at a higher rate (here $3 \mathrm{krpm}$ ) to give a sediment with slightly smaller nanosheets, which we 
label "2-3 krpm". The associated supernatant can be centrifuged again and the cascade continued for as many steps as are required with each step using a continually increasing centrifugation rate. Critically, because the heavier, few-layer nanosheets are removed in each step of the cascade, the resultant supernatants become more and more monolayer enriched. After each step, the sediment contains smaller and smaller nanosheets, resulting in effective size selection. Within this cascade, we terminate the cascade at $10 \mathrm{krpm}$ and only partially analyze the final supernatant as it mostly contained free surfactant and extremely small $\mathrm{WS}_{2}$ nanosheets. This process is illustrated in Figure 1. One very important feature of LCC is that virtually no material is wasted, resulting in the collection of relatively large masses of nanosheets in each fraction (see below).

Importantly, the cascade can be designed according to the desired outcome. Here, we wanted to produce a range of dispersions with varying nanosheet sizes and therefore we performed a set of centrifugations with subsequently increasing rpm. However, if only a specific size distribution is desired, the procedure can be simplified by trapping the desired nanosheets between two fixed rpm. For example, if the stock is directly centrifuged at $7.5 \mathrm{krpm}$ and the resultant supernatant then centrifuged at $10 \mathrm{krpm}$, the collected sediment will contain nanosheets with a size distribution virtually identical to those obtained from the "7.5-10 krpm" step in a cascade. Thus a cascade can be used when a set of different nanosheet sizes is required but only two centrifugations are required to obtain a single size-selected sample.

We have characterized the nanosheets collected in each fraction microscopically using both transmission electron microscopy (TEM) and atomic force microscopy (AFM) with typical images displayed in Figures 2 A-D (see supplementary Figures S1-S4). In order to characterize the size-selection of LCC, the nanosheet length (i.e. the longest dimension) was measured over all fractions. Example histograms are shown in Figures $2 \mathrm{E}$ and $2 \mathrm{~F}$ (see supplementary Figures S1, 4) for the 1.5-2 krpm and 7.5-10 krpm samples. These histograms show a reduction in nanosheet length as the centrifugation rates are increased (i.e. as the dispersion progresses through the cascade).

Along with nanosheet length and width (defined as the dimension perpendicular to length), the nanosheet thickness, expressed as number of monolayers per nanosheet, $\mathrm{N}$, was measured by AFM with the effect of residual surfactant corrected for using step height analysis (SI figure S5-6). ${ }^{9,15,26,31}$ Typical layer number histograms are shown in Figures 2G-H (see supplementary Figure S4). Again, we see a reduction in nanosheet thickness as the dispersion 
progresses through the cascade. Interestingly, the $\mathrm{N}$ histograms are always log-normal in shape (see SI section 1 1.1-1 1.2) as previously observed for LPE nanosheets. ${ }^{49}$

We can quantify these effects by plotting the mean nanosheet length (measured from TEM and AFM) as a function of the centrifugal acceleration ( $g$-force, see methods) associated with the midpoint of the pair of rpms used in each step of the cascade (see Figure 2I). The mean nanosheet length falls off as ( $g$-force $)^{-0.5}$ as expected due to the close relationship between $\langle L\rangle$ and the "cut size" (i.e. the size of the largest particle remaining dispersed after centrifugation, see supplementary Figure S7). ${ }^{21}$ The lateral dimensions measured by AFM are over-estimated due to tip broadening and pixilation effects. The data in Figure 2I has been used to correct the lateral dimensions measured by AFM for the rest of the analysis (see supplementary Figure S8). We note that we have also analyzed the very small nanosheets that are discarded in the final supernatant of the cascade. TEM (figure S2) confirms that these are indeed 2D nanosheets with $\langle\mathrm{L}\rangle \sim 25 \mathrm{~nm}$. Due to the small lateral dimensions, a reliable AFM analysis was not possible.

Similarly, the mean nanosheet thickness, $\langle N\rangle$, as measured by AFM is plotted versus $g$-force in Figure 2J. The mean number of layers falls with central rotational speed via ( $g$ force $)^{-0.4}$. For the set of size-selected samples studied here, it is clear from this data that $\langle L\rangle \propto\langle N\rangle$. This is certainly a limitation of this method as, ideally, one would like to vary $\langle L\rangle$ and $\langle N\rangle$ independently. Below and in the SI (figure S9) we discuss the relationship between $L$ and $N$ in more detail. We find the distribution widths (i.e. the standard deviation: $\Delta L$ or $\Delta N$ ) to scale linearly with the mean for both $L$ and $N$ (see Figures $2 \mathrm{~K}-\mathrm{L}$ ) with slopes that are quite high $(\sim 50 \%)$. This is also not ideal as much smaller values of $\Delta N /\langle N\rangle$ and $\Delta L /\langle L\rangle$ will be required for most applications. In this work, we have not optimised the procedure to give reduced distribution widths. However, we believe that LCC is versatile enough for such optimisation to be achievable.

AFM analysis can also be used to assess the population of monolayers expressed as monolayer number fraction, $N_{\text {mono }} / N_{T}$. and plotted in Figure $3 \mathrm{~A}$ as a function of the central $g$ force. Interestingly, we find $N_{\text {mono }} / N_{T}$ to scale linearly with $g$-force as the monolayer content is enriched. After 7 steps in the cascade (i.e. at central acceleration of $\sim 7000 \mathrm{~g}$ ), values of $N_{\text {mono }} / N_{T}$ as high as $40 \%$ have been achieved. In Figure $3 \mathrm{~B}$ we plot $N_{m o n o} / N_{T} v s$. $\langle N\rangle$, finding a very sharp fall off, emphasizing the importance in minimizing the mean nanosheet thickness for ML enrichment. 
Another way to describe the monolayer content is via the monolayer volume fraction, $V_{f}$. This has been calculated from the AFM data using the knowledge of nanosheet length, $L$, width, $W$, and thickness, $t$ (which we define as $N \times 0.6 \mathrm{~nm}$ ) according to equation 1 .

$$
V_{f}=\frac{\sum_{m o n o} L W t}{\sum_{\text {All }} L W t}=\frac{\sum_{\text {mono }} L W}{\sum_{\text {All }} L W N}
$$

We find $V_{\mathrm{f}}$ to scale as $(g-\text { force })^{1.9}$, reaching $20 \%$ after 7 steps in the cascade (see Figure 3C). Because few-layer nanosheets are typically of higher volume than monolayers, $V_{f}$ $<N_{\text {mono }} / N_{T}$.

Knowledge of $V_{\mathrm{f}}$ for each fraction is extremely useful as it allows us to assess the mass of ML nanosheets produced in each step once the total $\mathrm{WS}_{2}$ mass is known. For each step in the cascade, we measured the dispersed $\mathrm{WS}_{2}$ mass using a combination of weighing and spectroscopy (see supplementary Figure S10). This data is plotted versus central $g$-force in Figure 3D. The stock dispersion ( $80 \mathrm{~mL}$ in volume, $\sim 150 \mathrm{mg}$ of exfoliated $\mathrm{WS}_{2}$ ) was separated into 7 fractions with the first four fractions containing 25-40 $\mathrm{mg}$ of $\mathrm{WS}_{2}$ nanosheets each, considerably more than achieved using comparable processes. For example, a multistep centrifugation procedure has reported graphene nanosheet quantities $<1 \mathrm{mg}^{43}$ while density gradient ultracentrifugation has yielded $\mathrm{WS}_{2}$ monolayer-rich dispersions with concentrations of $<0.005 \mathrm{~g} / \mathrm{L} .{ }^{46}$

However, after the fourth step in the cascade, (i.e. for central $g$-forces above $\sim 2000 g$ ) the mass produced falls off sharply, reaching $\sim 1 \mathrm{mg}$ after step $7(\sim 7000 \mathrm{~g})$. The mass of monolayers produced is related to the total $\mathrm{WS}_{2}$ mass by $M_{M L}=V_{f} M_{T}$ allowing us to calculate the mass of monolayers as also plotted versus central $g$-force in Figure 3D. We find the monolayer mass to increase over the first 4 steps of the cascade as the dispersions become more and more enriched. However, even though enrichment continues in subsequent steps, it falls off after step 4 as the total dispersed mass declines. This data clearly shows that highly enriched samples can be achieved by collecting nanosheets using high centrifugation rates but at the cost of low yield. Alternatively, the mass of monolayers collected can be optimized by using midrange centrifugation rates.

Relationship between nanosheet length and thickness 
The data in figure $1 \mathrm{I}-\mathrm{J}$ shows the mean nanosheet length and thickness of the different samples to be in proportion to each other. However, this hides important trends within each sample. Shown in supplementary figure S9A is a plot of $L$ versus $N$ measured for all individual nanosheets over the seven samples prepared in the cascade described above. While this graph appears to be consistent with the proportionality of $L$ and $N$, closer inspection shows that, for a given sample (e.g. 4-6 krpm as shown in inset), the nanosheet length does not vary systematically with thickness. To investigate this more carefully, we extracted the mean nanosheet length for each value of $N$, which we plotted versus $N$ or each sample in figure S9B. This shows that for a given step in the cascade, the mean nanosheet length is roughly independent of $\mathrm{N}$ : e.g. for the 7.5-10 krpm sample, the mean monolayer length is $41 \mathrm{~nm}$ while the mean 4-layer length is $45 \mathrm{~nm}$. This implies that the separation mechanism predominately involves nanosheet length rather than mass as might be expected. However, lower central gforces result in larger nanosheets in general and so larger monolayers. For the relatively low central g-forces (2-3 krpm sample) the mean monomer length was as high as $130 \mathrm{~nm}$ with individual monolayers as long as $200 \mathrm{~nm}$ observed. Unfortunately, because these long monolayers are found in the low g-force samples, they tend to be relatively rare. To date we have not found a way to highly enrich a sample with long monolayers rather than the shorter $(\sim 50 \mathrm{~nm})$ monolayers found in the 7.5-10 krpm sample. We note that monolayer length distribution in the stock is probably set by the exfoliation conditions (e.g. details of sonication regime and stabilizer). In the future, it would be useful to identify conditions which would give a much larger initial population of large monolayers.

\section{Dependence of the optical extinction spectra on the nanosheet dimensions}

It has previously been shown in the case of liquid-exfoliated $\mathrm{MoS}_{2}$, that the spectral profile of optical extinction (or absorbance) spectra strongly depends on nanosheets dimensions due to edge and confinement effects. ${ }^{15}$ Here we use the fractions produced by LCC to investigate the effect of nanosheet size and thickness on the optical properties of LPE $\mathrm{WS}_{2}$.

To do this, we first measured the optical extinction spectra for the fractions described above (N.B. the extinction, Ext, is related to the transmittance, $T$, by $T=10^{-E x t}$ and to the extinction coefficient, $\varepsilon$, by $E x t=\varepsilon C l$ where $C$ is the nanosheet concentration and $l$ is the cell length). The extinction coefficients were calculated using the measured mass of dispersed nanosheets (see supplementary Figure S10). As shown in Figure 4A, optical extinction spectra of liquid-exfoliated $\mathrm{WS}_{2}$, display the characteristic excitonic transitions, ${ }^{27}$ but vary 
systematically with nanosheet size and thickness due to edge and confinement effects. ${ }^{15}$ Similar behavior is observed for the absorbance and scattering coefficient spectra (see supplementary Figure S11). ${ }^{15}$ Importantly, the scattering coefficients are relatively small for these nanosheets, meaning that the absorbance and extinction are very similar. The edge effects result in a dependence of the spectral profile on nanosheet length. As a result the extinction coefficient, for example at the A-exciton $(\sim 620 \mathrm{~nm})$, depends strongly on nanosheet length as shown in Figure 4B. However, the extinction coefficient at $235 \mathrm{~nm}$ is invariant with nanosheet length $\left(\varepsilon_{235 \mathrm{~nm}}=47.7 \mathrm{Lg}^{-1} \mathrm{~cm}^{-1}\right)$, allowing its universal use to measure the concentration of $\mathrm{WS}_{2}$.

The effect of edges on the spectral shape can be quantified via the ratio of extinction intensities at two different wavelengths e.g. at $235 \mathrm{~nm}$ to that at $290 \mathrm{~nm}$, Ext $_{235} /$ Ext $_{290}$, which is plotted in Figure 4C. Similar to $\mathrm{MoS}_{2}$, the data in Figure 4C can be fitted to the following equation $^{15}$

$$
\frac{\operatorname{Ext}_{235}}{\operatorname{Ext}_{290}}=\frac{\alpha_{C}(235 \mathrm{~nm}) L+2 x(k+1) \Delta \alpha(235 \mathrm{~nm})}{\alpha_{C}(290 \mathrm{~nm}) L+2 x(k+1) \Delta \alpha(290 \mathrm{~nm})}
$$

where $\alpha_{C}$ is the absorption coefficient associated with the nanosheet basal plane, $\Delta \alpha=\alpha_{E}-\alpha_{C}$ where $\alpha_{E}$ is the edge region absorption coefficient, and $\mathrm{L}, \mathrm{x}$ and $\mathrm{k}$ are the nanosheet length, edge thickness and aspect ratio respectively. ${ }^{15} \mathrm{We}$ find this equation fits the data very well allowing us to generate a function which relates the mean nanosheet length, $L$ to the extinction peak intensity ratio:

$$
L(n m)=\frac{2.3-E x t_{235} / E x t_{290}}{0.02 E x t_{235} / E x t_{290}-0.0185}
$$

This relationship is extremely useful as it allows the mean nanosheet length in any dispersion to be extracted from an extinction spectrum. Other peak intensity ratios yield similar relationships as discussed in the SI (see supplementary Figure S12).

In addition, the extinction spectra also contain information on mean nanosheet thickness due to confinement effects. These result in shifts of the A-exciton position (see Figure 4A inset) towards lower wavelengths as the nanosheet thickness is reduced. Interestingly, for the 7.5-10 krpm sample, previously unseen structure begins to appear with a peak at $\sim 612 \mathrm{~nm}$ and a shoulder at $\sim 622 \mathrm{~nm}$. The origin of this will be discussed in more detail below.

The relationship between the center of mass position of the A-exciton, $\lambda_{\mathrm{A}}$, (determined from the second derivative of the A-exciton, supplementary Figure S13) and the mean $\mathrm{WS}_{2}$ 
nanosheet thickness is displayed in Figure 4D. As with $\mathrm{MoS}_{2}, \lambda_{\mathrm{A}}$ increases logarithmically with nanosheet thickness. ${ }^{15} \mathrm{We}$ find data for $\mathrm{WS}_{2}$ exfoliated in sodium dodecylbenzene sulfonate (SDBS) and poly(vinyl alcohol) (PVA) to sit very close to the same line suggesting solvatochromic effects to be small (but not nonexistent, see SI). ${ }^{50}$

In any case, fitting the data in Figure 4D to an empirical relation gives an equation, which allows us to extract the mean nanosheet thickness from the wavelength associated with the Aexciton:

$$
\langle N\rangle=6.35 \times 10^{-32} e^{\lambda_{A}(n m) / 8.51}
$$

This equation is at least applicable to aqueous SC-, SDBS- and PVA-stabilized $\mathrm{WS}_{2}$ dispersions and almost certainly gives approximate nanosheet thicknesses in a wide range of liquid environments (see below and SI). Because these shifts in A-exciton position will become important later in the paper, we need to verify this behavior as much as possible. We found similar trends had been observed by Zhu et al. and Zhao et al. for absorbance/reflectance spectra of micromechanically cleaved $\mathrm{WS}_{2} .{ }^{51,52} \mathrm{We}$ note that the literature data from micromechanically cleaved $\mathrm{WS}_{2}$ is offset to lower energies compared to our data presumably to the different dielectric environment. ${ }^{50,53}$ In addition, we have attempted to confirm these effects by calculating the electronic band structure of $\mathrm{WS}_{2}$ nanosheets as a function of $\mathrm{N}$. As shown in Figure 4E, the computed direct transition at the K-point (see section: methods) of the Brillouin zone follows a similar logarithmic dependence on layer number as observed in the experimental data.

It is worth noting that equations 3 and 4 might only be expected to hold for surfactant exfoliated $\mathrm{WS}_{2}$ nanosheets. To test this we performed a cascade for $\mathrm{WS}_{2}$ nanosheets exfoliated in the solvent $\mathrm{N}$-methyl-pyrrolidone (NMP). ${ }^{4}$ We found the length metric described by equation 3 to apply very well to NMP-exfoliated $\mathrm{WS}_{2}$. However, we observed a small degree of solvatochromism which resulted in shifts in $\lambda_{\mathrm{A}}$ due to environmental effects $(\sim 1.5 \mathrm{~nm}$ shift between NMP- and SC-stabilized nanosheets). This means that although equation 4 can be used to approximately find nanosheet thicknesses from extinction spectra of NMP-based dispersions, slight modifications are required to accurately extract nanosheet thicknesses in NMP. This modified metric is given in Figure S23.

Dependence of the photoluminescence on the monolayer content in the dispersions 
While quantitative spectroscopic metrics for length and thickness are instructive, a metric for ML content would be even more useful. The most obvious candidate is the nanosheet photoluminescence (PL) as this is only appreciable in monolayers for the common group VITMDs. ${ }^{51,54-56}$ However, previous experiments on $\mathrm{MoS}_{2}$ dispersions showed that PL can only be detected using standard luminescence spectrometers for dispersions with high monolayer content, ${ }^{15}$ making routine PL characterization impossible.

Here we use the superior sensitivity of a Raman spectrometer to detect PL even in noncentrifuged stock dispersions with low ML content. We found that, when acquiring a Raman spectrum in liquid drops $\left(\lambda_{\mathrm{exc}}=532 \mathrm{~nm}\right)$, the photoluminescence of the monolayer is typically detected at high wavenumbers $\left(\sim 2460 \mathrm{~cm}^{-1}\right)$. N.B. the measurement has to be carried out with extreme care (see methods). The Raman/PL spectra normalized to the $\mathrm{WS}_{2} 2 \mathrm{LA}(\mathrm{M})$ Raman mode $\left(355 \mathrm{~cm}^{-1}, 543 \mathrm{~nm}\right)$ of size-selected $\mathrm{WS}_{2}$ nanosheet dispersions are plotted in Figure $5 \mathrm{~A}$ as a function of wavelength (also compare figure $\mathrm{S} 14$ ). The $\mathrm{WS}_{2}$ Raman modes plotted versus wavenumber are shown in the inset. In addition to the $\mathrm{WS}_{2}$ Raman modes, in all cases, the typical photoluminescence peak of the $\mathrm{WS}_{2}$ is detected at $\sim 612 \mathrm{~nm}$ (the feature at $\sim 650 \mathrm{~nm}$ is the Raman response of water).

A typical PL spectrum measured in this way is plotted versus photon energy in Figure 5B. All spectra fit reasonably well to single Lorentzians, representing excitonic emission. While we initially expected inhomogeneous broadening to render Gaussian fitting more appropriate, we consistently found more reliable fits using Lorentzians (see supplementary Figure S15). The Lorentzian is centered at $2.023 \mathrm{eV}$ invariant of centrifugation conditions (see Figure 5C inset). However, as shown in Figure 5C, the linewidth appears to fall off with increasing centrifugation rate suggesting larger nanosheets to be slightly more defective than smaller ones. It must be noted that these PL peaks are very narrow, displaying widths as low as $30 \mathrm{meV} . \mathrm{WS}_{2}$ usually displays PL line widths between 22 and $75 \mathrm{meV},{ }^{51,57}$ with the lower values found only on non-perturbing surfaces like BN. This suggests that, not only does the water/SC environment not significantly dope the nanosheets, but also that they are largely defect-free (see supplementary Figure S16).

As shown in Figure 5D, we found the ratio of PL to Raman intensity to increase strongly with central $g$-force as the monolayer population increases. This suggests that this intensity ratio, $I_{P L} / I_{\text {Raman }}$, can be quantitatively linked to the monolayer content. We propose that the intensity of the Raman spectrum is proportional to the total number of $\mathrm{WS}_{2}$ formula units probed by the laser. However, because only the monolayers are luminescent, the PL intensity 
should be proportional to only the $\mathrm{WS}_{2}$ formula units associated with monolayers. This implies that $I_{P L} / I_{\text {Raman }}$ should scale linearly with the monolayer volume fraction. To test this, in Figure $5 \mathrm{E}$ we plot $I_{P L} / I_{\text {Raman }}$ versus the monolayer volume fraction, $V_{f}$, (as measured by AFM, Figure 3C). We find a linear relationship which holds of $>2$ decades of both $I_{P L} / I_{\text {Raman }}$ and $V_{f}$. Interestingly, equivalent measurements for $\mathrm{WS}_{2}$ nanosheets exfoliated in SDBS and PVA give data which falls on the same curve strongly suggesting that this metric is quite robust towards changes in the stabilizer, even though it may depend on the quality of the $\mathrm{WS}_{2}$ starting material. To further test the robustness of this metric, as well as the metrics based on UV-Vis spectroscopy, we have redispersed $\mathrm{WS}_{2}$ size-selected by LCC in SC of varying concentrations from 0-5 g/L. As shown in figures S17-18, spectra and hence the results of the metric analysis do not change as a function of stabilizer concentration. In addition, this shows that the LCC size-selected $\mathrm{WS}_{2}$ can even be redispersed in water without aggregation occurring immediately.

From the fit line in Figure 5E, we find

$$
V_{f}=\frac{1}{17} \frac{I_{P L}}{I_{\text {Raman }}}
$$

This relationship holds over a broad range of sizes and monolayer contents, but we note that it eventually breaks down for very small nanosheets, as edges may activate non-radiative decay. A first indication that this is indeed the case is obtained from an analysis of the very small nanosheets that are discarded in the supernatant after centrifuging at $10 \mathrm{krpm}$ (see SI figure S14, $\langle L\rangle \sim 25 \mathrm{~nm}$ ). While TEM and optical extinction show no obvious deviation from larger nanosheets, the PL/Raman ratio is significantly lower than in the 7.5-10 krpm sample suggesting edge effects to play a role. This issue is addressed further in the supporting information.

Nonetheless, this is an important result, as it means that the monolayer volume fraction in a dispersion of $\mathrm{WS}_{2}$ nanosheets can be quantified very simply and easily from a Raman/PL measurement. This is hugely advantageous over traditional techniques such as AFM or TEM, which are very time consuming and can require skill and experience from the experimenter. Critically, it allows us to track the monolayer content as we perform further monolayer enrichment as described below.

Optical properties of dispersions highly enriched in monolayers 
In the preceding sections we described a very simple primary centrifugation cascade which resulted in the production of a range of size-selected fractions at much higher yields than homogeneous centrifugation (see supplementary Figure S19), the last of which was monolayerenriched up to $N_{\text {mono }} / N_{T} \sim 40 \%$ and $V_{f} \sim 20 \%$. However, the strength of LCC is its versatility. For example, it is also applicable to nanosheets exfoliated in solvents (see SI Figures S20-23). In addition, much more complex cascades can be designed, resulting in higher degrees of monolayer enrichment. For example, we find the monolayer content to rise rapidly with iteration cycle when repeating the centrifugation at fixed rpm (see supplementary Figure S24).

In the following, we used the ability to spectroscopically measure the volume fraction of monolayers to design secondary cascades with the goal of maximizing $I_{P L} / I_{\text {Raman }}$. Details are presented in the Supporting Information (see supplementary Figures S25-38). In brief, we designed centrifugation protocols for monolayer enrichment consisting of multiple iterations starting from size-selected dispersions. These typically involved centrifugation at relatively low speeds and longer times (overnight) to remove few-layered $\mathrm{WS}_{2}$ and centrifugation at high speeds and short times (1-4h) to remove very small nanosheets (see supplementary Figure S25). We note that when designing such a cascade, if highly luminescent dispersions are required, the small-nanosheet-removal step is crucial as we found PL/Raman ratios to be significantly lower for very small nanosheets even at high ML contents, probably due to edge effects (see supplementary Figures S33-S34).

Below we will describe secondary cascades (S.C.), which used strongly (6-8 krpm, S.C.1, Figure S26-28) and weakly (1.5-10 krpm, S.C.2, Figure S29-31) size-selected dispersions as starting points for monolayer enrichment. Shown in Figure 6A is an AFM layer number histogram for the S.C.2 dispersion with a typical image in the inset. It is clear that this sample is dominated by monolayers with AFM analysis giving $N_{\text {mono }} / N_{T} \sim 74 \%$ and $V_{f} \sim 70 \%$ (see table 1 for all monolayer content data). The Raman/PL spectra of the 6-8 krpm dispersion and both ML enriched dispersions are shown in Figure 6B. While the 6-8 krpm dispersion displayed $I_{P L} / I_{\text {Raman }}=2.8$, equivalent to $V_{f}=0.16$, it is clear that the enriched samples display considerably more intense PL. We found $\mathrm{I}_{\mathrm{PL}} / \mathrm{I}_{\mathrm{Raman}}=10.9$ and 12.7, for the S.C.1 and S.C.2 dispersions, giving $V_{f}$ of $65 \%$ and $75 \%$, respectively, in good agreement with AFM statistics (Table 1).

Shown in Figure 6C are extinction spectra for both the 6-8 krpm sample and the S.C.1 and S.C.2 dispersions. The shapes of these spectra are almost indistinguishable because high speed centrifugation steps have been included in the cascade to remove very small nanosheets giving similar $\langle\mathrm{L}\rangle$ values for these dispersions (confirmed by AFM statistics, see 
supplementary Figure S28). We note that in this nanosheet size range, scattering ${ }^{15}$ is virtually non-existent so the extinction spectrum is identical to the absorbance spectrum (see supplementary Figure S33).

However, much more interesting is the shape of the A-excitonic components of the extinction spectra as shown in the inset (see Figure 4A inset). These show changes not only in center of mass peak position but also in shape. Unlike the spectra associated with thicker nanosheets, the 6-8 krpm spectrum clearly has a peak at $\sim 612 \mathrm{~nm}$ and a shoulder at $\sim 622 \mathrm{~nm}$ as discussed above (see Figure 4A). However, the ML-rich samples clearly have A-excitonic responses, which are dominated by a peak close to $610 \mathrm{~nm}(2.032 \mathrm{eV})$. This is extremely close to the position of the A-excitonic PL peak (2.023 eV, implying a Stokes shift of $10 \mathrm{meV}$ ). Thus we associate the feature at $\sim 610 \mathrm{~nm}$ in the extinction/absorbance spectrum of the ML-rich sample with the absorbance of monolayer $\mathrm{WS}_{2}$. This suggests the component at $\sim 622 \mathrm{~nm}$ represents the combined contribution of few-layered $\mathrm{WS}_{2}$.

To test this in more detail, we carefully smoothed the extinction spectra of all samples (see SI section 7) before finding the second derivative with respect to energy, $\mathrm{d}^{2}\left(\right.$ Ext)/dE ${ }^{2}$. Such a procedure is known to narrow contributing peaks roughly threefold, allowing resolution of closely spaced peaks. ${ }^{58}$ Samples of the resultant second derivatives are shown in Figure 6D. Monolayer-poor samples such as 1.5-2 krpm are dominated by one peak at $\sim 1.98 \mathrm{eV}$ while the monolayer-rich sample (S.C.1) is dominated by one peak at $\sim 2.03 \mathrm{eV}$, with intermediate samples showing both components.

We can extract more information about the components by fitting the second derivatives. In the simplest form, a Lorentzian line can be described by

$$
L(E)=\frac{h}{\left[1+\left(\frac{\left(E-E_{0}\right)}{w / 2}\right)^{2}\right]}
$$

where $h$ represents the height, $E_{0}$ the centre and $w$ the FWHM. As described in the SI (see SI section 5), we have fit the second derivative curves to the sum of two doubly differentiated Lorentzians, allowing us to extract height, width and position for both (see supplementary eq. S10 and Figure S39). As before, Lorentzians gave better fits than Gaussians (see Figures S4041). We find extremely good fits in all cases (see solid lines in Figure 6D). The higher energy component always had a position of $\mathrm{E}_{0}=2.035 \mathrm{eV}$ consistent with it representing absorbance of the monolayer A-exciton. The lower energy component was found between 1.98 and $1.997 \mathrm{eV}$. 
We associate this component with the individually unresolvable sum of few-layer A-exciton absorptions. In fact, the monolayer component can be differentiated from the few-layers only because of the logarithmic dependence embodied in equation 4 . This equation implies that the energy difference between A-excitonic transitions for 1- and 2- layer nanosheets is $19 \mathrm{meV}$ while being only $11 \mathrm{meV}$ between 2- and 3-layer nanosheets and $8 \mathrm{meV}$ between 3 - and 4layer nanosheets.

If the mono- and few-layer assignment described above is correct, then we expect the width of the monolayer A-exciton absorbance peak to be invariant with the width of the nanosheet thickness distribution. Conversely, the width of the few-layer A-exciton absorbance peak should increase as the thickness distribution broadens. This is exactly what we observed as plotted in Figure 6E (the lines are guides to the eye). The FL peak width increases from $~ 65$ to $\sim 110 \mathrm{meV}$ as the AFM thickness histogram width increases from 1-4.5. Conversely the monolayer peak is always $\sim 55 \mathrm{meV}$ wide. Interestingly this is almost twice as wide as the PL peak. This likely partly stems from the fact that all monolayered nanosheets are probed in absorbance, while the smallest nanosheets are not captured in the PL measurement (see supplementary Figures S23-S24).

With this peak assignment in mind, we propose that the area under the ML A-exciton extinction peak should scale with the monolayer content in the dispersion. Because the area under any Lorentzian is proportional to $h \times w$, we have calculated the metric $\mathrm{S}_{\mathrm{A}}$ :

$$
S_{A}=\frac{h_{M L} w_{M L}}{h_{M L} w_{M L}+h_{F L} w_{F L}}
$$

which we suggest, should scale with the monolayer volume fraction. We have plotted $S_{A}$ versus $V_{f}$ in Figure $6 \mathrm{~F}$, finding good linearity as described by $S_{A}=(0.8 \pm 0.05) V_{f}$. The fact that the proportionality constant is so close to 1 is a strong indicator that our assignment is correct. This allows us to use $S_{A}$ as an alternative metric for the monolayer volume fraction:

$V_{f}=(1.25 \pm 0.08) S_{A}$

The availability of highly monolayer-enriched dispersions allows us to measure the photoluminescence using a standard PL spectrometer exciting with Xe lamp to obtain excitation emission contour plots as shown in Figure 6G. Emission from the A-exciton can be well resolved with fitting of the emission spectrum (see supplementary Figure S32) giving position and widths virtually identical to those measured in the Raman spectrometer. 
The real advantage of measurement with the PL spectrometer is the ability to measure excitation spectra. Such spectra generally allow the measurement of the absorption spectrum of the luminescent species at higher resolution than would usually be possible. As shown in Figure $6 \mathrm{H}$, the measured excitation spectrum $\left(\lambda_{\mathrm{em}}=617 \mathrm{~nm}\right)$ shows the same spectral features as the extinction spectrum. However, these features, which can be attributed to B, C and D excitonic transitions, are considerably sharper in the excitation spectrum. As an inset in Figure 6I, we also compare the measured PL spectrum with the deconvoluted A-exciton absorbance contributions from mono-and few layer $\mathrm{WS}_{2}$ (calculated from the second derivative fit parameters). The monolayer A-exciton absorbance is very close to the PL with only a slight Stokes shift $(\sim 10 \mathrm{meV})$.

To test our understanding of this behavior, we have used $a b$ initio GW plus BetheSalpeter method to calculate the absorption spectrum of both mono- and bi-layer $\mathrm{WS}_{2}$ as shown in Figure 6I (see section methods). We find a strong dependence on layer number with the Aexciton shifting from $2.06 \mathrm{eV}$ for the ML to $1.99 \mathrm{eV}$ in the bilayer in reasonable agreement with the data in Figure 4D. We can model the absorption spectrum of a monolayer rich dispersion (S.C.2) by calculating the weighted average of the theoretical mono- and bilayer spectra where the weighting factors are the measured mono- and bilayer volume fractions (see Figure 6A, we ignore the small tri-layer population for simplicity). We find excellent agreement between measured and calculated spectra in the A-exciton regime once the calculated spectra have been normalized and downshifted by $25 \mathrm{meV}$. We attribute the shift to a combination of environmental and temperature effects.

\section{Conclusion}

Liquid cascade centrifugation is a simple, powerful and broadly applicable technique to separate liquid exfoliated nanosheets by size. It has a number of advantages over other techniques; notably its high yield and lack of wastage; the ability to control the concentration of size-selected suspensions, even up to very high concentrations. Probably most important is its versatility: cascades can be designed to produce the desired size and thickness distributions and the required degree of monolayer enrichment. Ultimately, we believe cascades will be designed to produce dispersions containing only a given nanosheet thickness at a predetermined lateral size. In addition, this technique can be applied to virtually any $2 \mathrm{D}$ material stabilized by solvents, surfactants or polymers using only benchtop centrifuges. 
The ability to easily size-select nanosheets has enabled us to study their optical properties as a function of size and thickness. Similar to $\mathrm{MoS}_{2},{ }^{15}$ we found the extinction spectra of $\mathrm{WS}_{2}$ suspensions to contain quantitative information describing both mean length and thickness of the nanosheets while the PL spectra quantify the monolayer content. Spectroscopic measurement of size, thickness and monolayer content allows cascade design to achieve further monolayer enrichment, enabling the study of the fundamental optical properties of nanosheet ensembles. For example, once the monolayer content was increased beyond $\mathrm{N}_{\text {mono }} / \mathrm{N}_{\mathrm{T}} \sim 25 \%$, fine structure began to appear in the extinction spectra in the vicinity of the A-exciton. We found it possible to differentiate the contributions to A-exciton extinction of mono- and few-layer nanosheets allowing us to use a simple extinction spectrum to measure the monolayer content of a suspension.

We believe that the ability to easily size select and monolayer enrich dispersions, coupled with the availability of quantitative spectroscopic metrics to assess mean nanosheet length, thickness and monolayer volume fraction will have impact for both applications and fundamental studies. Nanosheet sizes and thicknesses will be precisely tailored according to needs in samples, which will be available in large quantities. This will be especially critical for applications such as printed electronics where dispersions of electronically identical, and so uniformly sized, nanosheets are required. Optical analysis will become a tool for quality control allowing the concentration, size, thickness and monolayer content to be assessed for any dispersion. In addition, PL will be used as a fingerprint, which is sensitive to doping and environmental effects allowing an in situ probe for intermolecular interactions.

\section{Methods}

Sample preparation: $\mathrm{WS}_{2}$ dispersions were prepared by probe sonicating the powder. $\mathrm{WS}_{2}$ $(20 \mathrm{~g} / \mathrm{L})$ was immersed in $80 \mathrm{~mL}$ of aqueous surfactant solution $\left(\mathrm{C}_{\text {surf }}=6 \mathrm{~g} / \mathrm{L}\right)$. The mixture was sonicated under ice-cooling in a metal beaker by probe sonication using a solid flathead tip (Sonics VX-750) for $1 \mathrm{~h}$ at $60 \%$ amplitude. The dispersion was centrifuged in a Hettich Mikro 220R centrifuge equipped with a fixed-angle rotor 1016 at $5 \mathrm{krpm}(2660 \mathrm{~g})$ for $1.5 \mathrm{~h}$. The supernatant was discarded and the sediment collected in $80 \mathrm{~mL}$ of fresh surfactant and subjected to a second sonication $5 \mathrm{~h}$ at $60 \%$ amplitude with a pulse of $6 \mathrm{~s}$ on and $2 \mathrm{~s}$ off.

To select nanosheets by size, we used controlled centrifugation with sequentially increasing rotation speeds. Two different rotors were used (see SI). In the standard primary 
cascade, unexfoliated $\mathrm{WS}_{2}$ was removed by centrifugation at $1.5 \mathrm{krpm}(240 \mathrm{~g}, 2 \mathrm{~h})$. The supernatant was subjected to further centrifugation at $2 \mathrm{krpm}(426 g, 2 \mathrm{~h})$. The sediment was collected in fresh surfactant at reduced volume (3-8 mL), while the supernatant was centrifuged at $3 \mathrm{krpm}(958 \mathrm{~g}, 2 \mathrm{~h})$. Again, the sediment was collected and the supernatant subjected to centrifugation at higher speeds. This procedure was repeated with the following speeds: $4 \mathrm{krpm}$ (1700 g, 2 h), 5 krpm (2660 g, 2 h), 6 krpm (3506 g, 2 h), 7.5 krpm (5480 g, 2 h), 10 krpm $(9740 \mathrm{~g}, 2 \mathrm{~h})$. The data presented in Figure 1 uses the central $\mathrm{rpm} / \mathrm{g}$-force to express the consecutive centrifugation. For example, the sediment collected from the centrifugation between 2-3 krpm has a central rpm of $2.5 \mathrm{krpm}(665 \mathrm{~g})$.

To perform the monolayer enrichment, a sample size-selected by the standard procedure was subjected to further iterative centrifugation steps. Details are described in Section 4 of the Supplementary Information. The sample of S.C.1 shown in Figure 6 was produced from a standard size selection between 6-8 krpm. The dispersion was then centrifuged at $4 \mathrm{krpm}(1560$ $g$ ) for $6 \mathrm{~h}$. the sediment was discarded and the supernatant centrifuged at $5 \mathrm{krpm}(2435 \mathrm{~g})$ for $14 \mathrm{~h}$. The sediment was discarded and the supernatant centrifuged at $9 \mathrm{krpm}(7890 \mathrm{~g}, 4 \mathrm{~h})$. Very small nanosheets were removed in the supernatant after further centrifugation at $15 \mathrm{krpm}$ (21915 g, $1 \mathrm{~h})$. Alternatively, for S.C.2 a dispersion containing nanosheets sedimenting between 1.5-10 krpm was centrifuged as follows: $2.5 \mathrm{krpm}(609 \mathrm{~g}, 16 \mathrm{~h})$, supernatant subjected to $4 \mathrm{krpm}(1560 \mathrm{~g}, 14 \mathrm{~h})$, supernatant subjected to $10 \mathrm{krpm}(9740 \mathrm{~g}, 1 \mathrm{~h})$, sediment collected in $1.5 \mathrm{~mL}$ and subjected to $5 \mathrm{krpm}(2436 \mathrm{~g}, 5 \mathrm{~h})$, supernatant subjected to $8 \mathrm{krpm}(6235 \mathrm{~g}, 2$ h), sediment collected and subjected to $3 \mathrm{krpm}(877 \mathrm{~g}, 12 \mathrm{~h})$. The supernatant after this last centrifugation step was collected and had a monolayer volume fraction of $\sim 70-75 \%$.

Characterization: Optical extinction was measured on a Varian Cary 500 in quartz cuvettes 1 $\mathrm{nm}$ increments. Bright field transmission electron microscopy imaging on Holey carbon grids (400 mesh) was performed using a JEOL 2100, operated at $200 \mathrm{kV}$. Statistical analysis was performed of the flake dimensions by measuring the longest axis of the nanosheet and assigning it "length" and the dimension perpendicular to the longest axis which we defined as "width". Atomic force microscopy (AFM) was carried out on a Veeco Nanoscope-IIIa (Digital Instruments) system in tapping mode after depositing a drop of the dispersion $(10 \mu \mathrm{L})$ on a preheated $\left(150{ }^{\circ} \mathrm{C}\right) \mathrm{Si} / \mathrm{SiO}_{2}$. The apparent thickness was converted to number of layers using previously elaborated step-height analysis of liquid-exfoliated nanosheets. ${ }^{15}$ Raman and photoluminescence spectroscopy was performed on the liquid dispersions using a Horiba Jobin Yvon LabRAM HR800 with $532 \mathrm{~nm}$ excitation laser in air under ambient conditions. Great 
care must be taken during these measurements, as changes in the focal plane during the acquisition will introduce an error in the PL/Raman ratio (see SI methods). A drop ( $\sim 40 \mu \mathrm{L})$ of a high concentration dispersion was placed on a glass slide and the drop edge was optically focused using a $10 \times$ objective. The focus for the measurement with the $100 \times$ objective was readjusted in such a way that the laser was focused slightly above the drop. The average of $\sim 5$ measurements are displayed. Photoluminescence to obtain the contour plot and excitation spectra was measured in quartz cuvettes using an Edinburgh Instruments FS920 PL spectrometer equipped with a Xe lamp $(450 \mathrm{~W})$ and a S900 photomultiplier tube detector at room temperature with single monochromators in excitation and emission.

\section{Theoretical Optical Gap Calculations:}

The optical gap is extracted from the optical conductivity calculated by using the tightbinding propagation method (TBPM). ${ }^{59,60} \mathrm{We}$ adopt an 11-band TB model of few-layered $\mathrm{WS}_{2}$ proposed by R. Roldán et al in Ref. ${ }^{61}$, consisting with five d orbitals of $\mathrm{W}$ atom and six $\mathrm{p}$ orbitals of $\mathrm{S}$ atom as the follows:

W atoms: $d_{3 z^{2}-r^{2}}, d_{x^{2}-y^{2}}, d_{x y}, d_{x z}, d_{y z}$,

$\mathrm{S}$ atoms: $p_{x, t}, p_{y, t}, p_{z, t}, p_{x, b}, p_{y, b}, p_{z, b}$,

where the $t$ and $b$ indexes indicate the top and bottom planes of $\mathrm{S}$ atoms within the same layer, respectively. The Slater-Koster parameters used to construct the intralayer W-S, W-W and S$\mathrm{S}$ hopping matrixes are (in unit of eV) $\Delta_{0}=-0.872, \Delta_{1}=0.42, \Delta_{2}=-2.065, \Delta_{p}=-3.468$, $\Delta_{z}=-3.913, V_{p d \sigma}=3.603, V_{p d \pi}=-0.942, V_{d d \sigma}=-1.216, V_{d d \pi}=0.177, V_{d d \delta}=$ $0.243, V_{p p \sigma}=0.749, V_{p p \pi}=0.236$, and for the interlayer S-S hoppings are $U_{p p \sigma}=-0.55$, $U_{p p \pi}=-0.6$. The spin-orbital couplings originating from the $\mathrm{W}$ and $\mathrm{S}$ atoms are $\lambda_{W}=0.215$, $\lambda_{S}=0.057$.

The real part of the optical conductivity at finite frequency is calculated via the Kubo's formula as ${ }^{59-61}$

$$
\sigma(\omega)=\lim _{\varepsilon \rightarrow+0} \frac{1-e^{-\beta \hbar \omega}}{\hbar \omega \Omega} \int_{0}^{\infty} d t e^{i(\omega+i \varepsilon) \tau} 2 i \operatorname{Im}\langle\phi|J[1-f(H)] J(\tau) f(H)| \phi\rangle,
$$

where $\Omega$ is the sample area, $\beta=1 / k_{B} T$ is the inverse temperature, $H$ is the tight-binding Hamiltonian, $f(H)=1 /\{\exp [\beta(H-\mu)]+1\}$ is the Fermi-Dirac distribution operator, and $J(\tau)=\exp (i H \tau / \hbar) J \exp (-i H \tau / \hbar)$ is the current operator in the Heisenberg picture. The state 
$|\phi\rangle$ is a normalized random state which covers all the eigenstates in the whole spectrum. ${ }^{59}$ The time evolution operator and Fermi-Dirac distribution operator are represented as the Chebyshev polynomial expansions.

\section{$A b$ initio GW-BSE simulations:}

We start the optical calculations using the Kohn-Sham eigenvectors and energy eigenvalues previously calculated within the density-functional theory at generalized-gradient approximation, ${ }^{62}$ for monolayer and bilayer $\mathrm{WS}_{2}$, using plane waves basis set and periodic boundary conditions as implemented in the PWscf ${ }^{63}$ and $\operatorname{Vasp}^{64,65}$ codes. Norm-conserving ${ }^{66}$ and $\mathrm{PAW}^{67,68^{\prime}}$ pseudopotentials are used with a plane wave energy cutoff of $900 \mathrm{eV}$, with partial-core states included. Atomic coordinates were allowed to relax until all forces were smaller in magnitude than $0.01 \mathrm{eV} / \AA$. Relevant lattice constants (in-plane and out-of-plane) were optimized for each system. To avoid interactions between supercell images, the distance between periodic images of the $\mathrm{WS}_{2}$ layers along the direction perpendicular to the plane was always larger than $20 \AA$. Spin-orbit interactions are included in the calculations perturbatively through the calculations of the spinor wave functions, which are used as an input for the calculation of the dielectric functions $\varepsilon_{2}(\omega, q)$ afterwards. The GW-BSE calculations are done using the Yambo code ${ }^{69}$ using 300 unoccupied bands in the integration of the self-energy term. The number of $k$-points was chosen according to the Monkhorst-Pack scheme ${ }^{70}$ and was set to the equivalent of a $21 \times 21 \times 1$ grid in the primitive unit cell of $\mathrm{WS}_{2}$, which was previously converged for all structures. The BSE Hamiltonian was created using the ten highest valence bands and the six lowest conduction bands using the Tamm-Dancoff approach. The response functions were obtained in a fine grid of 1000 energy points using a broadening of $0.04 \mathrm{eV}$ in all calculations.

Supporting Information Available: Detailed methods, materials characterisation and description of a range of cascades. This material is available free of charge via the Internet at http://pubs.acs.org.

\section{Acknowledgement}

The research leading to these results has received funding from the European Union Seventh Framework Program under grant agreement $n^{\circ} 604391$ Graphene Flagship. We have also 
received support from the Science Foundation Ireland (SFI) funded centre AMBER (SFI/12/RC/2278). In addition, JNC acknowledges the European Research Council (SEMANTICS) and SFI (11/PI/1087) for financial support. CB acknowledges the German research foundation DFG (BA 4856/1-1). VV-M and CG acknowledge Marie Curie ITN network "MoWSeS" (grant no. 317451). EJGS acknowledges the use of computational resources provided by the Extreme Science and Engineering Discovery Environment (XSEDE), supported by NSF grants number TG-DMR120049, TG-DMR150017; as well as the Queen's Fellow Award through the startup grant number M8407MPH. We thank David McAteer for help preparing figure 1. 


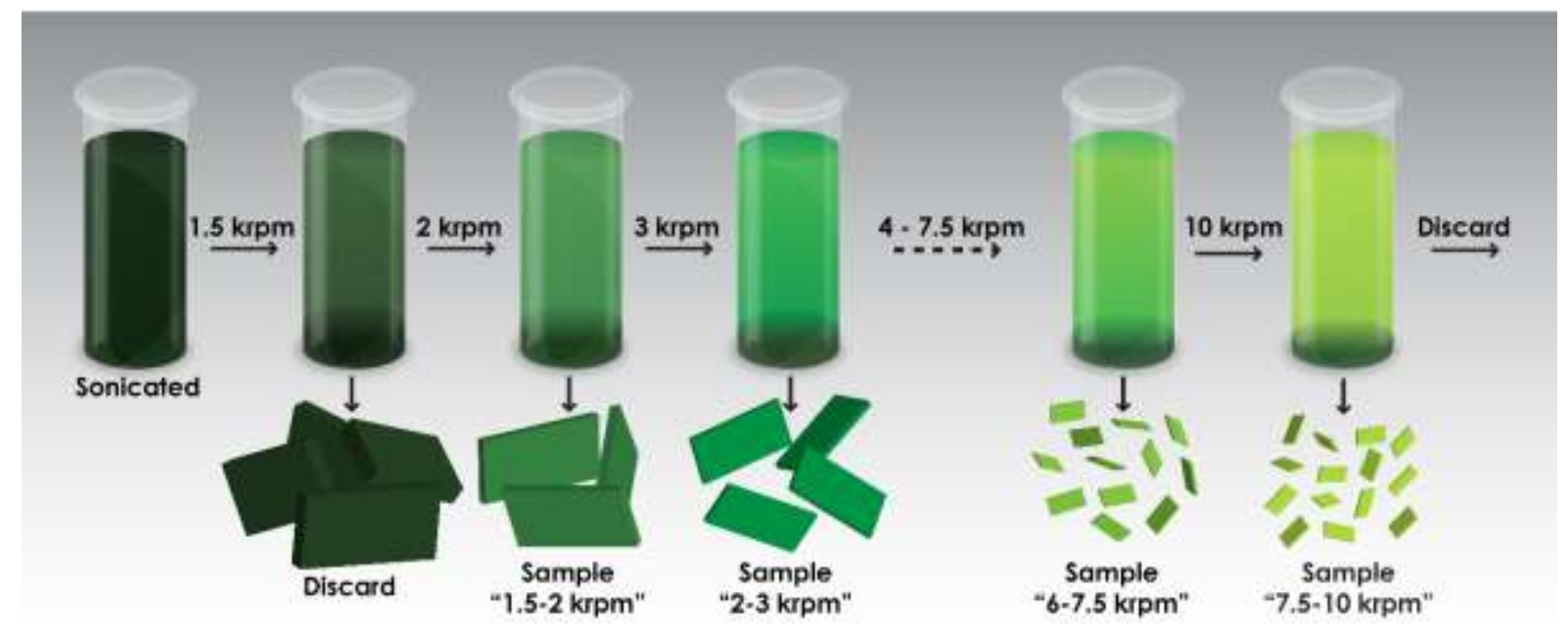

Figure 1: Schematic describing the basic centrifugation cascade employed in this study. The sediment discarded after the first centrifugation contains exfoliated layered crystallites while the supernatant discarded after the last centrifugation step contains extremely small nanosheets. Size-selected dispersions are prepared by re-dispersing the collected sediments in $1 \mathrm{~g} / \mathrm{L}$ aqueous sodium cholate after subsequently increasing centrifugation speeds. 

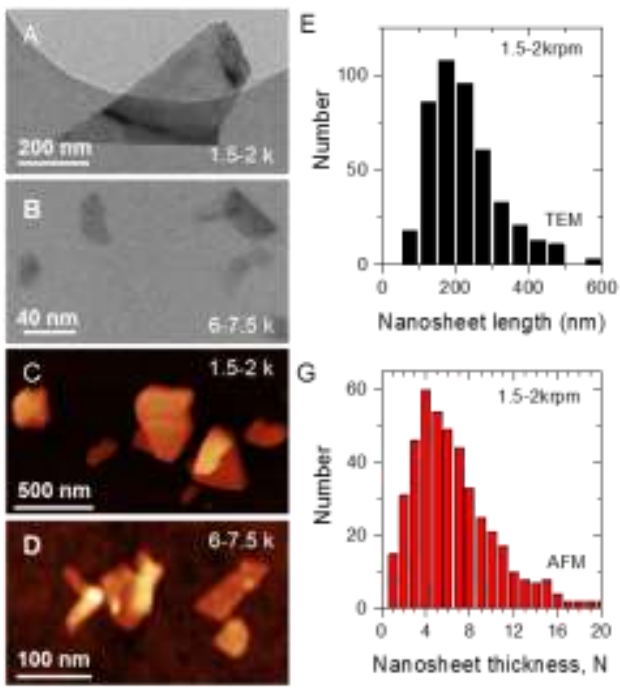
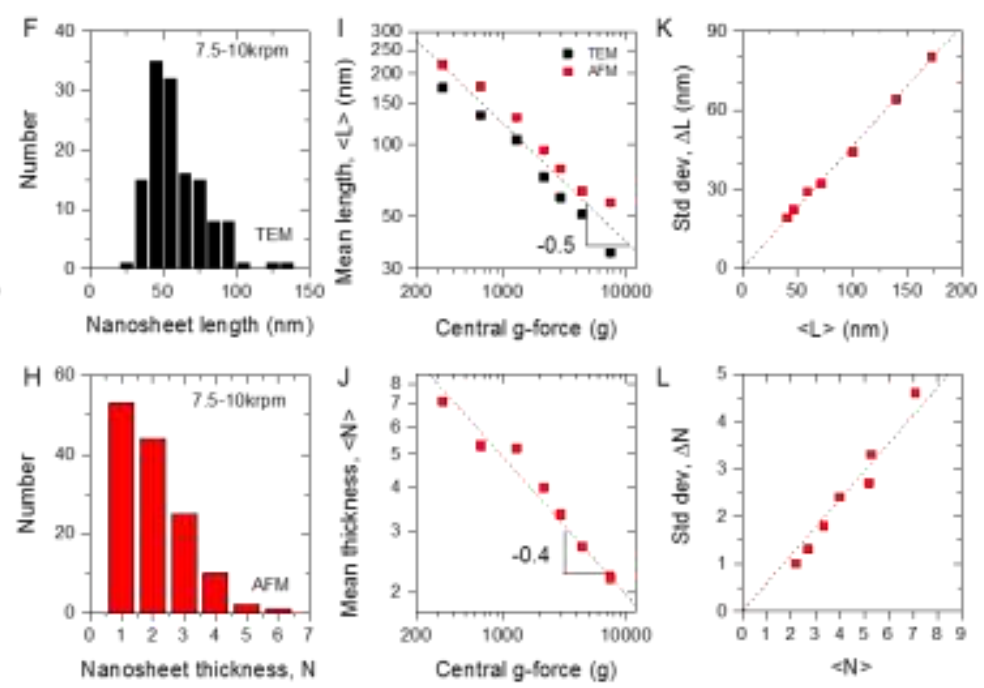

Figure 2: Microscopic characterization of size-selected $\mathrm{WS}_{2}$ nanosheets. A-D) TEM (A-B) and AFM (C-D) images of nanosheets, size-selected with upper and lower centrifugation speeds of (A\&C) $1.5 \mathrm{krpm}$ and $2 \mathrm{krpm}$ and (B\&D) $6 \mathrm{krpm}$ and $7.5 \mathrm{krpm}$ respectively. E-J) Statistical analysis of dimensions of nanosheets extracted from images such as those in A-D. This is presented as histograms representing data for lateral nanosheet size measured by TEM (E-F) and nanosheet thickness (number of monolayers per nanosheet, $\mathrm{N}$ ) determined by AFM (G-H). Data is shown for two combinations of centrifugation speed, 1.5-2 krpm and 6-7.5 krpm. I, J) Data extracted from histograms for a range of centrifugation conditions. Mean values of I) nanosheet length, $\langle L\rangle$, and $\mathrm{J}$ ) nanosheet thickness, $\langle N\rangle$, as a function of the central centrifugation acceleration, presented as central $g$-force. K-L) Standard deviation of nanosheet length $(\mathrm{K})$ and thickness $(\mathrm{L})$ plotted versus mean length and thickness respectively. The dashed lines in $\mathrm{K}$ and $\mathrm{L}$ represent $\Delta L /\langle L\rangle=0.46$ and $\Delta N /\langle N\rangle=0.59$ respectively. 

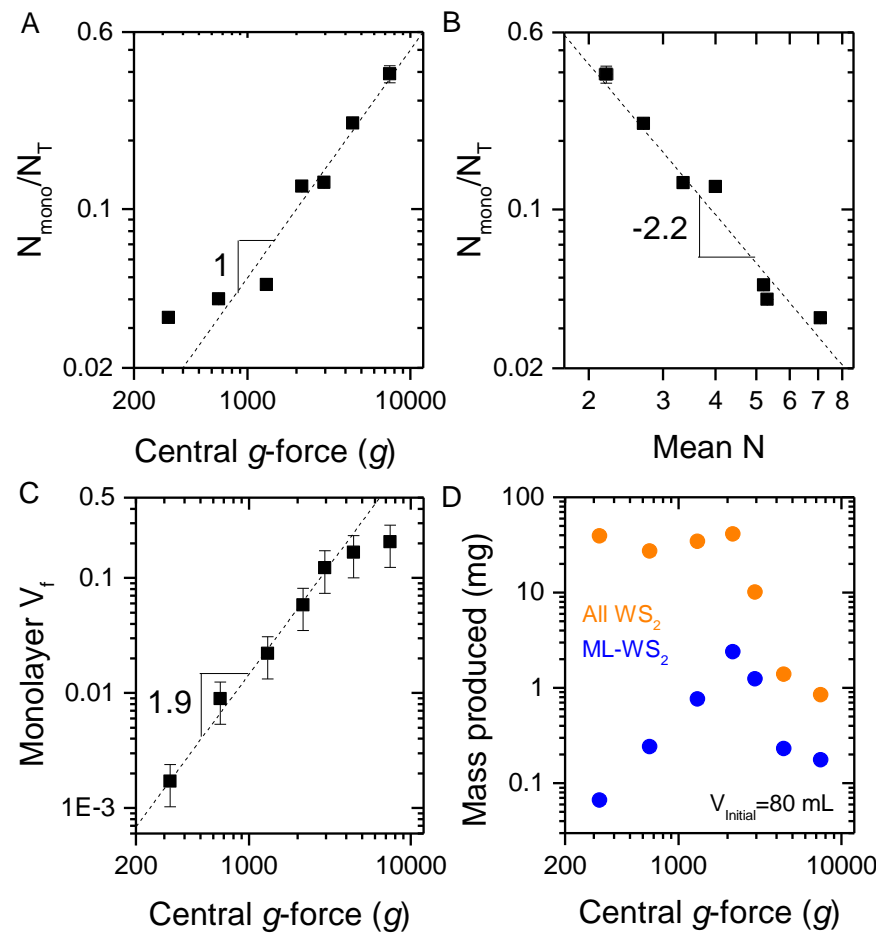

Figure 3: Monolayer population data extracted from histograms for a range of centrifugation conditions. A) Number fraction of monolayers, $\mathrm{N}_{\text {mono }} / \mathrm{N}_{\mathrm{T}}$, as a function of the central centrifugation acceleration, presented as $g$-force. B) Plot of $\mathrm{N}_{\text {mono }} / \mathrm{N}_{\mathrm{T}}$ versus mean nanosheet thickness, N. C) Monolayer volume fraction as a function of $g$-force. D) Total mass of all $\mathrm{WS}_{2}$ as well as only monolayer-WS 2 produced in each fraction as a function of $g$-force. 

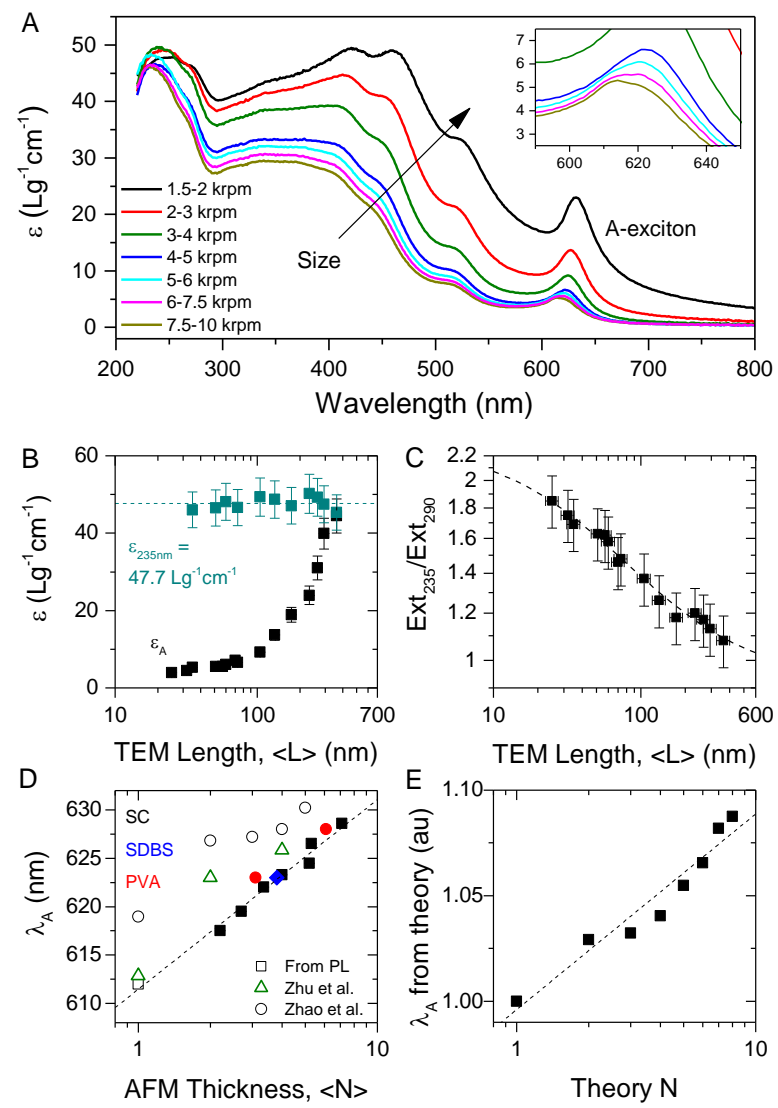

Figure 4: Dependence of the optical properties of nanosheet dispersions on the nanosheet dimensions. A) Optical extinction coefficient spectra measured for $\mathrm{WS}_{2}$ dispersions (water/SC) prepared using different centrifugation conditions, and so with different mean nanosheet lengths and thicknesses. Inset: magnified A-exciton region. B) Extinction coefficient, measured at $235 \mathrm{~nm}$ and at the A-exciton position $(\sim 615 \mathrm{~nm})$ plotted versus mean nanosheet length, as measured by TEM. The mean value of $\varepsilon_{235}$ is $47.7 \mathrm{Lg}^{-1} \mathrm{~cm}^{-1}$. C) Ratio of extinction at $235 \mathrm{~nm}$ to that at $290 \mathrm{~nm}$ plotted versus mean nanosheet length, as measured by TEM. The dashed line is a fit to eq. 2. D) A-exciton center of mass position (determined from second derivatives) plotted versus mean nanosheet thickness. Also included is data for $\mathrm{WS}_{2}$ nanosheets dispersed in SDBS and PVA and from the literature. ${ }^{51,52}$ The open symbol represents the Aexciton position from photoluminescence measurements. The dashed line shows an empirical relationship between $\lambda_{A}$ and $\mathrm{N}$ according to eq. 4 . E) Calculated relative wavelength associated with the optical gap of $\mathrm{WS}_{2}$ (i.e. the direct band gap at the K-point). 

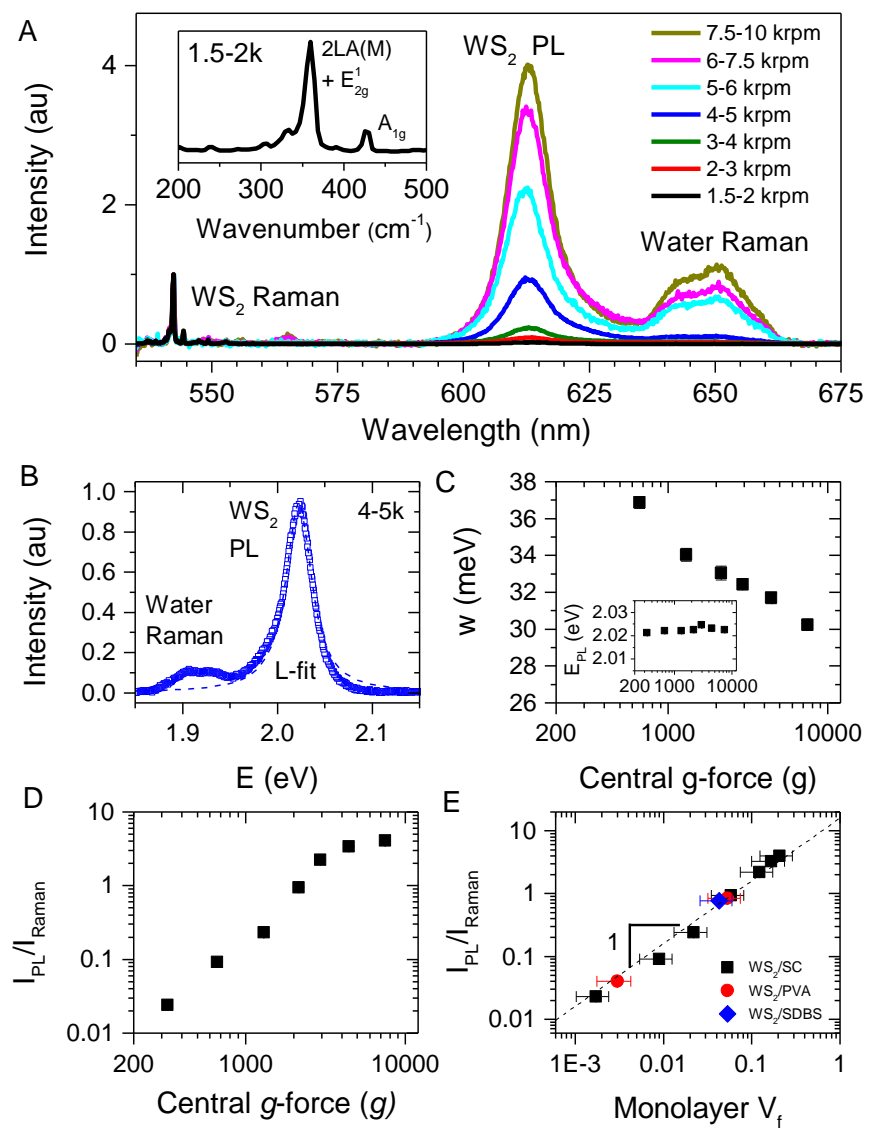

Figure 5: Photoluminescence data for dispersions with different monolayer content. A) Photoluminescence $(\sim 610 \mathrm{~nm})$ and Raman $(\sim 540 \mathrm{~nm})$ spectra of surfactant-stabilized $\mathrm{WS}_{2}$ dispersions prepared with different centrifugation conditions and measured in liquid using a Raman spectrometer $\left(\lambda_{\text {exc }}=532 \mathrm{~nm}\right)$. The spectra were normalized to the $2 \mathrm{LA}(\mathrm{M})$ Raman mode of $\mathrm{WS}_{2}$. The feature at $\sim 650 \mathrm{~nm}$ is the water Raman peak. Inset: Raman spectrum of dispersed $\mathrm{WS}_{2}$ nanosheets plotted versus wavenumber. B) Photoluminescence spectrum of $\mathrm{WS}_{2}$, plotted on an energy scale and fitted to a Lorentzian. C) PL linewidth, from Lorentzian fit, plotted versus central centrifugation acceleration (expressed in units of $g$ ). Inset: PL position vs. $g$ force. D) Ratio of PL intensity to Raman intensity, $\mathrm{I}_{\mathrm{PL}} / \mathrm{I}_{\mathrm{Raman}}$, plotted $v s$. $g$-force. E) $\mathrm{I}_{\mathrm{PL}} / \mathrm{I}_{\mathrm{Raman}}$ plotted versus monolayer volume fraction. The dashed line represents $I_{P L} / I_{\text {Raman }}=17 \times V_{f}$. 

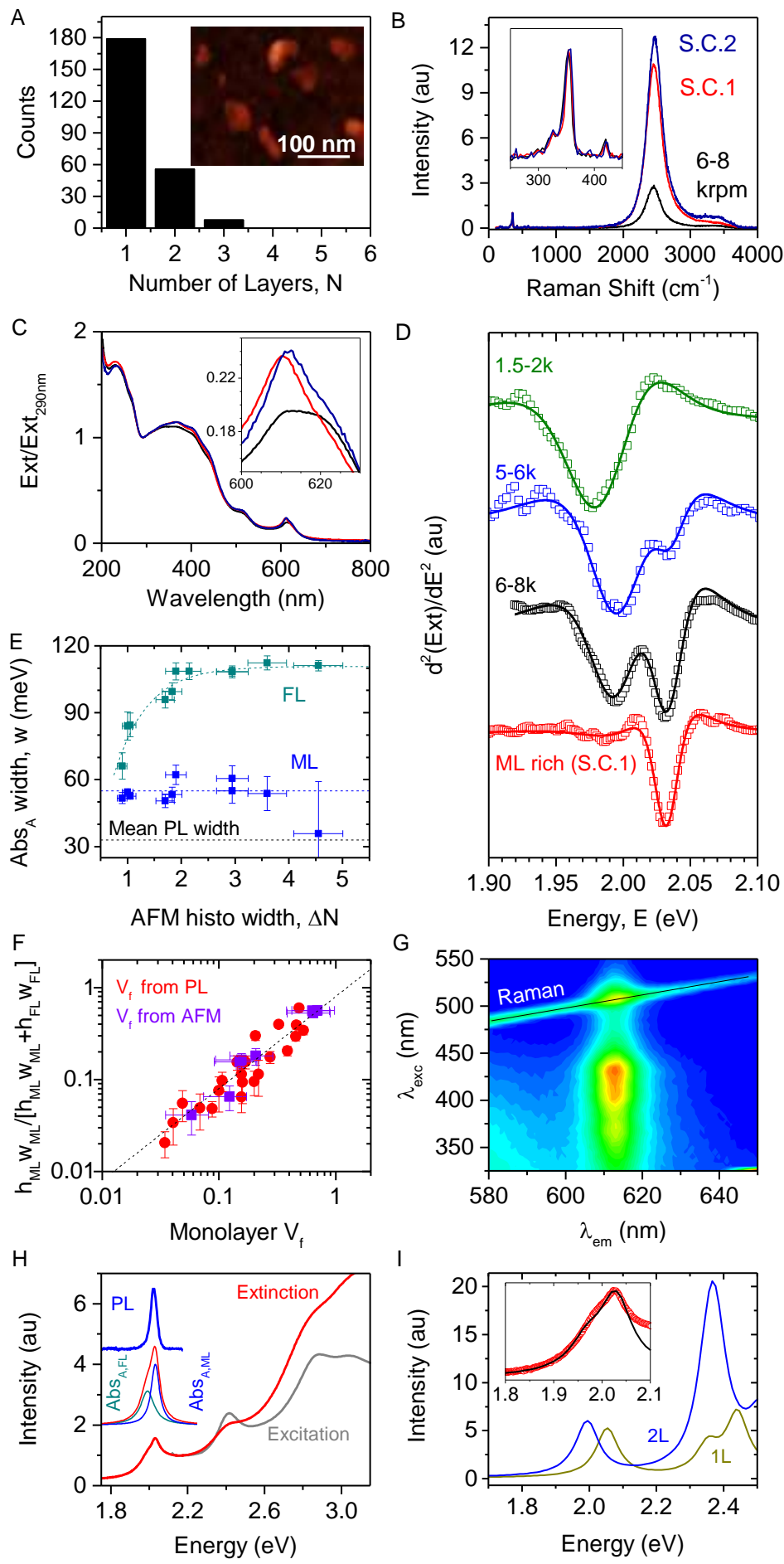

Figure 6: Characterization of second stage monolayer enriched dispersions. A) AFM nanosheet thickness (expressed as layer number) histogram and representative image of $\mathrm{WS}_{2}$ nanosheets from a dispersion enriched in monolayers by a refined LCC. B) Raman/PL spectra of the ML-rich dispersion (red) compared to the first stage size-selected dispersion used for the ML enrichment centrifugation (blue). An alternative secondary cascade yielded a similar dispersion (yellow). C) Optical extinction spectra of the three dispersion. Inset: Zoom in of the 
A-exciton showing clear changes in spectral shape. D) Second derivative spectra for a number of $\mathrm{WS}_{2}$-SC dispersions fitted to the sum of the second derivatives of two Lorentzians. With increasing ML volume fraction, a well separated component at 2.033(3) eV ( 610 nm) can clearly be identified which is attributed to $\mathrm{ML}^{-\mathrm{WS}_{2}}$. E) Width of absorbance Lorentzian curves, representing mono- and few-layer nanosheets, found by fitting second derivatives such as those shown in D plotted versus the full width at half maximum of the corresponding AFM thickness histogram. Shown for comparison is the mean width of the PL spectra in Figure 5A. F) Aexciton shape monolayer metric, obtained from fitting the second derivative of the extinction spectra to two Lorentzians, as a function of ML volume fraction. The violet squares represent data where $V_{\mathrm{f}}$ was measured from $A F M$, whereas $V_{\mathrm{f}}$ was determined from the PL/Raman ML metric in the case of the red data points. The dashed line shows a linear relation that can be used to determine the $V_{f}$ from the shape of the A exciton according to equation 8. G) Excitationemission contour plot of the ML-rich dispersion measured in a PL spectrometer. H) Extinction spectrum of the ML-rich dispersion compared to the excitation spectrum at the ML emission (after subtraction of the water background). The same excitonic features are evident in both spectra. Lower inset: A-exciton absorbance deconvoluted into the individual components of $\mathrm{ML}$ and few-layer $\mathrm{WS}_{2}$ as well as their sum. Upper inset: Measured PL spectrum (450 nm excitation). I) Theoretical absorption curves for monolayer (1L) and bi-layer (2L) $\mathrm{WS}_{2}$. Inset: Extinction curve measured for S.C.2 monolayer enriched sample (open symbols). Also shown is the weighted sum $\left(A b s=0.71 A b s_{1 \mathrm{~L}}+0.24 A b s_{2 \mathrm{~L}}\right.$, black line $)$ of the theoretical monolayer and bilayer absorption spectrum. These weightings were chosen to reflect the volume fraction of monolayers and bilayers in the S.C.2 sample as measured by AFM (see Figure 6A) where we have neglected the small population of trilayers for simplicity. N.B the theory curve has been downshifted to $25 \mathrm{meV}$ to match the experimental data.

\begin{tabular}{|l|l|l|l|l|}
\hline Sample & $\langle\mathrm{N}\rangle$ & $\mathrm{N}_{\text {mono }} / \mathrm{N}_{\mathrm{T}}$ & $\mathrm{V}_{\mathrm{f}}$ & $\mathrm{I}_{\mathrm{PL}} / \mathrm{I}_{\text {Raman }}$ \\
\hline 6-8 krpm & 2.4 & 0.30 & 0.15 & 2.7 \\
\hline S.C.1 & 1.6 & 0.73 & 0.64 & 10.8 \\
\hline $1.5-10 \mathrm{krpm}$ & 9.0 & 0.04 & 0.01 & 0.18 \\
\hline S.C. 2 & 1.6 & 0.74 & 0.70 & 12.7 \\
\hline
\end{tabular}

Table 1: Summary of data relating to monolayer content for size-selected starting dispersions and dispersions enriched by secondary cascades. 


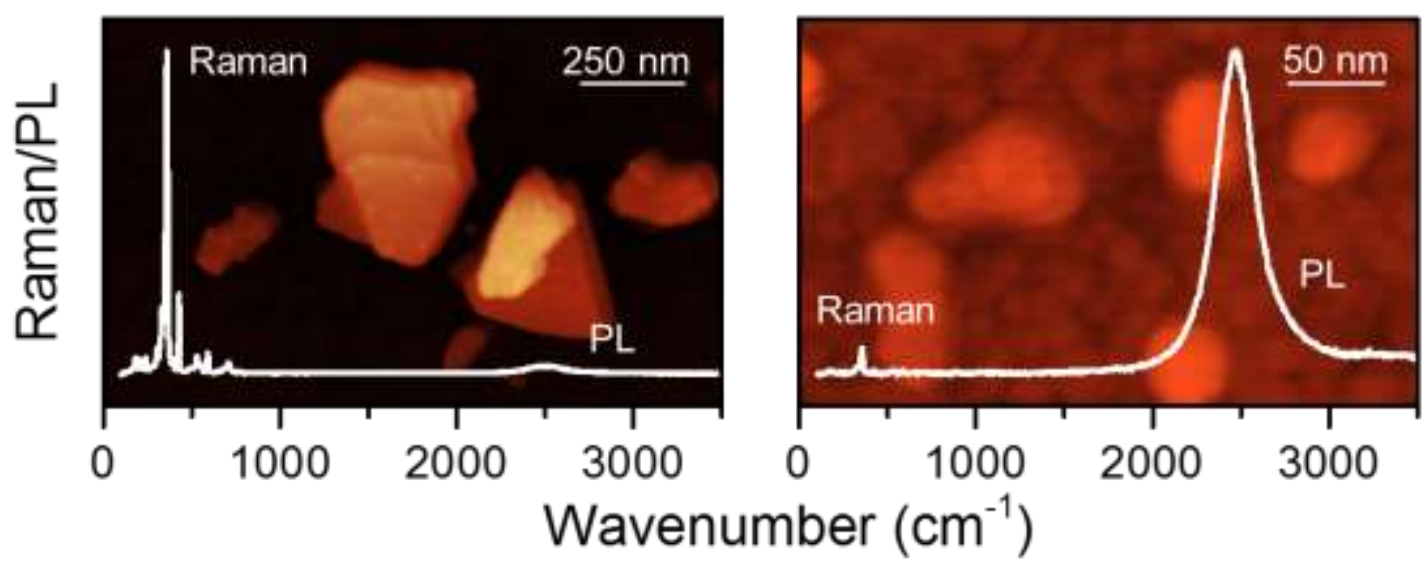

\section{References}

1. Nicolosi, V.; Chhowalla, M.; Kanatzidis, M. G.; Strano, M. S.; Coleman, J. N., Liquid Exfoliation of Layered Materials. Science 2013, 340, 1420-+.

2. Hernandez, Y.; Nicolosi, V.; Lotya, M.; Blighe, F. M.; Sun, Z.; De, S.; McGovern, I. T.; Holland, B.; Byrne, M.; Gun'Ko, Y. K., et al., High-Yield Production of Graphene by Liquid-Phase Exfoliation of Graphite. Nat. Nanotechnol. 2008, 3, 563-568.

3. Green, A. A.; Hersam, M. C., Solution Phase Production of Graphene with Controlled Thickness Via Density Differentiation. Nano Lett. 2009, 9, 4031-4036.

4. $\quad$ Coleman, J. N.; Lotya, M.; O'Neill, A.; Bergin, S. D.; King, P. J.; Khan, U.; Young, K.; Gaucher, A.; De, S.; Smith, R. J., et al., Two-Dimensional Nanosheets Produced by Liquid Exfoliation of Layered Materials. Science 2011, 331, 568-571.

5. Zhi, C. Y.; Bando, Y.; Tang, C. C.; Kuwahara, H.; Golberg, D., Large-Scale Fabrication of Boron Nitride Nanosheets and Their Utilization in Polymeric Composites with Improved Thermal and Mechanical Properties. Adv. Mater. 2009, 21, 2889-+.

6. Bourlinos, A. B.; Georgakilas, V.; Zboril, R.; Steriotis, T. A.; Stubos, A. K., Liquid-Phase Exfoliation of Graphite Towards Solubilized Graphenes. Small 2009, 5, 1841-1845.

7. Ciesielski, A.; Samori, P., Graphene Via Sonication Assisted Liquid-Phase Exfoliation. Chem. Soc. Rev. 2014, 43, 381-398.

8. Varrla, E.; Backes, C.; Paton, K. R.; Harvey, A.; Gholamvand, Z.; McCauley, J.; Coleman, J. N., Large-Scale Production of Size-Controlled Mos2 Nanosheets by Shear Exfoliation. Chem. Mater. 2015, 27, 1129-1139.

9. $\quad$ Paton, K. R.; Varrla, E.; Backes, C.; Smith, R. J.; Khan, U.; O’Neill, A.; Boland, C.; Lotya, M.; Istrate, O. M.; King, P., et al., Scalable Production of Large Quantities of Defect-Free Few-Layer Graphene by Shear Exfoliation in Liquids. Nat. Mater. 2014, 13, 624-630.

10. Hernandez, Y.; Lotya, M.; Rickard, D.; Bergin, S. D.; Coleman, J. N., Measurement of Multicomponent Solubility Parameters for Graphene Facilitates Solvent Discovery. Langmuir 2010, 26, 3208-3213.

11. Cunningham, G.; Lotya, M.; Cucinotta, C. S.; Sanvito, S.; Bergin, S. D.; Menzel, R.; Shaffer, M. S. P.; Coleman, J. N., Solvent Exfoliation of Transition Metal Dichalcogenides: Dispersibility of Exfoliated Nanosheets Varies Only Weakly between Compounds. ACS Nano 2012, 6, 3468-3480.

12. Lotya, M.; Hernandez, Y.; King, P. J.; Smith, R. J.; Nicolosi, V.; Karlsson, L. S.; Blighe, F. M.; De, S.; Wang, Z. M.; McGovern, I. T., et al., Liquid Phase Production of Graphene by Exfoliation of Graphite in Surfactant/Water Solutions. J. Am. Chem. Soc. 2009, 131, 3611-3620.

13. Smith, R. J.; King, P. J.; Lotya, M.; Wirtz, C.; Khan, U.; De, S.; O'Neill, A.; Duesberg, G. S.; Grunlan, J. C.; Moriarty, G., et al., Large-Scale Exfoliation of Inorganic Layered Compounds in Aqueous Surfactant Solutions. Adv. Mater. 2011, 23, 3944-3948. 
14. Wu, M.; Wang, Y.; Lin, X.; Yu, N.; Wang, L.; Wang, L.; Hagfeldt, A.; Ma, T., Economical and Effective Sulfide Catalysts for Dye-Sensitized Solar Cells as Counter Electrodes. Phys. Chem. Chem. Phys. 2011, 13, 19298-301.

15. Backes, C.; Smith, R. J.; McEvoy, N.; Berner, N. C.; McCloskey, D.; Nerl, H. C.; O’Neill, A.; King, P. J.; Higgins, T.; Hanlon, D., et al., Edge and Confinement Effects Allow in Situ Measurement of Size and Thickness of Liquid-Exfoliated Nanosheets. Nature Commun. 2014, 5, 4576.

16. May, P.; Khan, U.; Hughes, J. M.; Coleman, J. N., Role of Solubility Parameters in Understanding the Steric Stabilization of Exfoliated Two-Dimensional Nanosheets by Adsorbed Polymers. J. Phys. Chem. C 2012, 116, 11393-11400.

17. Liang, Y. T.; Hersam, M. C., Highly Concentrated Graphene Solutions Via Polymer Enhanced Solvent Exfoliation and Iterative Solvent Exchange. J. Am. Chem. Soc. 2010, 132, 17661-17663.

18. Khan, U.; May, P.; O'Neill, A.; Bell, A. P.; Boussac, E.; Martin, A.; Semple, J.; Coleman, J. N., Polymer Reinforcement Using Liquid-Exfoliated Boron Nitride Nanosheets. Nanoscale 2013, 5, 581-587.

19. Bourlinos, A. B.; Georgakilas, V.; Zboril, R.; Steriotis, T. A.; Stubos, A. K.; Trapalis, C., Aqueous-Phase Exfoliation of Graphite in the Presence of Polyvinylpyrrolidone for the Production of Water-Soluble Graphenes. Solid State Commun. 2009, 149, $2172-2176$.

20. Coleman, J. N., Liquid Exfoliation of Defect-Free Graphene. Acc. Chem. Res. 2013, 46, 14-22.

21. Nacken, T. J.; Damm, C.; Walter, J.; Ruger, A.; Peukert, W., Delamination of Graphite in a High Pressure Homogenizer. RSC Adv. 2015, 5, 57328-57338.

22. Zhou, K. G.; Mao, N. N.; Wang, H. X.; Peng, Y.; Zhang, H. L., A Mixed-Solvent Strategy for Efficient Exfoliation of Inorganic Graphene Analogues. Angew. Chem.,Int. Ed. 2011, 50, 10839-10842.

23. Bang, G. S.; Nam, K. W.; Kim, J. Y.; Shin, J.; Choi, J. W.; Choi, S. Y., Effective Liquid-Phase Exfoliation and Sodium Ion Battery Application of Mos2 Nanosheets. ACS Appl. Mater. Interfaces 2014, 6, 7084-7089.

24. Alsaif, M.; Balendhran, S.; Field, M. R.; Latham, K.; Wlodarski, W.; Ou, J. Z.; Kalantar-Zadeh, K., Two Dimensional Alpha-Moo3 Nanoflakes Obtained Using Solvent-Assisted Grinding and Sonication Method: Application for H-2 Gas Sensing. Sens. Actuators, B 2014, 192, 196-204.

25. Hanlon, D.; Backes, C.; Higgins, T. M.; Hughes, M.; O’Neill, A.; King, P.; McEvoy, N.; Duesberg, G. S.; Mendoza Sanchez, B.; Pettersson, H., et al., Production of Molybdenum Trioxide Nanosheets by Liquid Exfoliation and Their Application in High-Performance Supercapacitors. Chem. Mater. 2014, 26, 1751-1763.

26. Harvey, A.; Backes, C.; Gholamvand, Z.; Hanlon, D.; McAteer, D.; Nerl, H. C.; McGuire, E.; Seral-Ascaso, A.; Ramasse, Q. M.; McEvoy, N., et al., Preparation of Gallium Sulfide Nanosheets by Liquid Exfoliation and Their Application as Hydrogen Evolution Catalysts. Chem. Mater. 2015, 27 , 3483-3493.

27. Yasaei, P.; Kumar, B.; Foroozan, T.; Wang, C.; Asadi, M.; Tuschel, D.; Indacochea, J. E.; Klie, R. F.; Salehi-Khojin, A., High-Quality Black Phosphorus Atomic Layers by Liquid-Phase Exfoliation. Adv. Mater. 2015, 27, 1887-1892.

28. Vishnu Sresht; Agilio A.H. Padua; Blankschtein, Liquid-Phase Exfoliation of Phosphorene: Design Rules from Molecular Dynamics Simulations. ACS Nano 2015, DOI: 10.1021/acsnano.5b02683.

29. Kang, J.; Wood, J. D.; Wells, S. A.; Lee, J.-H.; Liu, X.; Chen, K.-S.; Hersam, M. C., Solvent Exfoliation of Electronic-Grade, Two-Dimensional Black Phosphorus. ACS Nano 2015, 9, 3596-3604. 30. Brent, J. R.; Savjani, N.; Lewis, E. A.; Haigh, S. J.; Lewis, D. J.; O'Brien, P., Production of Few-Layer Phosphorene by Liquid Exfoliation of Black Phosphorus. Chem. Commun. 2014, 50, 1333813341.

31. Hanlon, D.; Backes, C.; Doherty, E.; Cucinotta, C. S.; Berner, N. C.; Boland, C.; Lee, K.; Lynch, P.; Gholamvand, Z.; Harvey, A., et al., Liquid Exfoliation of Solvent-Stabilised Few-Layer Black Phosphorus for Applications Beyond Electronics. Nature Commun. 2015, 6, 8563.

32. Naguib, M.; Mashtalir, O.; Carle, J.; Presser, V.; Lu, J.; Hultman, L.; Gogotsi, Y.; Barsoum, M. W., Two-Dimensional Transition Metal Carbides. ACS Nano 2012, 6, 1322-1331.

33. Zhou, K.; Jiang, S.; Bao, C.; Song, L.; Wang, B.; Tang, G.; Hu, Y.; Gui, Z., Preparation of Poly(Vinyl Alcohol) Nanocomposites with Molybdenum Disulfide (Mos2): Structural Characteristics and Markedly Enhanced Properties. RSC Adv. 2012, 2, 11695-11703. 
34. Paton, K. R.; Varrla, E.; Backes, C.; Smith, R. J.; Khan, U.; O'Neill, A.; Boland, C.; Lotya, M.; Istrate, O. M.; King, P., et al., Scalable Production of Large Quantities of Defect-Free Few-Layer Graphene by Shear Exfoliation in Liquids. Nat. Mater. 2014, 13, 624-30.

35. Eksik, O.; Gao, J.; Shojaee, S. A.; Thomas, A.; Chow, P.; Bartolucci, S. F.; Lucca, D. A.; Koratkar, N., Epoxy Nanocomposites with Two-Dimensional Transition Metal Dichalcogenide Additives. ACS Nano 2014, 8, 5282-5289.

36. Sorrentino, A.; Altavilla, C.; Merola, M.; Senatore, A.; Ciambelli, P.; Iannace, S., Nanosheets of Mos2-Oleylamine as Hybrid Filler for Self-Lubricating Polymer Composites: Thermal, Tribological, and Mechanical Properties. Polym. Compos. 2015, 36, 1124-1134.

37. May, P.; Khan, U.; O'Neill, A.; Coleman, J. N., Approaching the Theoretical Limit for Reinforcing Polymers with Graphene. J. Mater. Chem. 2012, 22, 1278-1282.

38. Wang, J.-Z.; Lu, L.; Lotya, M.; Coleman, J. N.; Chou, S.-L.; Liu, H.-K.; Minett, A. I.; Chen, J., Development of Mos2-Cnt Composite Thin Film from Layered Mos2 for Lithium Batteries. Advanced Energy Materials 2013, 3, 798-805.

39. Pumera, M.; Sofer, Z.; Ambrosi, A., Layered Transition Metal Dichalcogenides for Electrochemical Energy Generation and Storage. J. Mater. Chem. C. 2014, 2, 8981-8987.

40. Woodward, R. I.; Kelleher, E. J. R.; Howe, R. C. T.; Hu, G.; Torrisi, F.; Hasan, T.; Popov, S. V.; Taylor, J. R., Tunable Q-Switched Fiber Laser Based on Saturable Edge-State Absorption in FewLayer Molybdenum Disulfide (Mos2). Optics Express 2014, 22, 31113-31122.

41. Z Zhang, M.; Howe, R. T.; Woodward, R.; Kelleher, E. R.; Torrisi, F.; Hu, G.; Popov, S.; Taylor, J. R.; Hasan, T., Solution Processed Mos2-Pva Composite for Sub-Bandgap Mode-Locking of a Wideband Tunable Ultrafast Er:Fiber Laser. Nano Research 2015, 8, 1522-1534.

42. $\quad$ Sun, Z.; Hasan, T.; Torrisi, F.; Popa, D.; Privitera, G.; Wang, F.; Bonaccorso, F.; Basko, D. M.; Ferrari, A. C., Graphene Mode-Locked Ultrafast Laser. ACS Nano 2010, 4, 803-810.

43. Khan, U.; O'Neill, A.; Porwal, H.; May, P.; Nawaz, K.; Coleman, J. N., Size Selection of Dispersed, Exfoliated Graphene Flakes by Controlled Centrifugation. Carbon 2012, 50, 470-475.

44. Jaramillo, T. F.; Jorgensen, K. P.; Bonde, J.; Nielsen, J. H.; Horch, S.; Chorkendorff, I., Identification of Active Edge Sites for Electrochemical H-2 Evolution from Mos2 Nanocatalysts. Science 2007, 317, 100-102.

45. Torrisi, F.; Coleman, J. N., Electrifying Inks with 2d Materials. Nat. Nanotechnol. 2014, 9, 738739.

46. Kang, J.; Seo, J.-W. T.; Alducin, D.; Ponce, A.; Yacaman, M. J.; Hersam, M. C., Thickness Sorting of Two-Dimensional Transition Metal Dichalcogenides Via Copolymer-Assisted Density Gradient Ultracentrifugation. Nat Commun 2014, 5, 5478.

47. O'Neill, A.; Khan, U.; Coleman, J. N., Preparation of High Concentration Dispersions of Exfoliated Mos2 with Increased Flake Size. Chem. Mater. 2012, 24, 2414-2421.

48. Barwich, S.; Khan, U.; Coleman, J. N., A Technique to Pretreat Graphite Which Allows the Rapid Dispersion of Defect-Free Graphene in Solvents at High Concentration. J. Phys. Chem. C 2013, 117, 19212-19218.

49. Kouroupis-Agalou, K.; Liscio, A.; Treossi, E.; Ortolani, L.; Morandi, V.; Pugno, N. M.; Palermo, V., Fragmentation and Exfoliation of 2-Dimensional Materials: A Statistical Approach. Nanoscale 2014, 6, 5926-5933.

50. $\quad$ Lin, Y.; Ling, X.; Yu, L.; Huang, S.; Hsu, A. L.; Lee, Y.-H.; Kong, J.; Dresselhaus, M. S.; Palacios, T., Dielectric Screening of Excitons and Trions in Single-Layer Mos2. Nano Lett. 2014, 14, 5569-5576.

51. Zhao, W.; Ghorannevis, Z.; Chu, L.; Toh, M.; Kloc, C.; Tan, P.-H.; Eda, G., Evolution of Electronic Structure in Atomically Thin Sheets of Ws2 and Wse2. ACS Nano 2012, 7, 791-797.

52. Zhu, B.; Chen, X.; Cui, X., Exciton Binding Energy of Monolayer Ws2. Sci. Rep. 2015, 5.

53. Scheuschner, N.; Ochedowski, O.; Kaulitz, A.-M.; Gillen, R.; Schleberger, M.; Maultzsch, J., Photoluminescence of Freestanding Single- and Few-Layer Mos2. Phys. Rev. B 2014, 89, 125406.

54. Mak, K. F.; Lee, C.; Hone, J.; Shan, J.; Heinz, T. F., Atomically Thin Mos2. A New DirectGap Semiconductor. Phys. Rev. Lett. 2010, 105, 136805/1-136805/4.

55. Splendiani, A.; Sun, L.; Zhang, Y.; Li, T.; Kim, J.; Chim, C.-Y.; Galli, G.; Wang, F., Emerging Photoluminescence in Monolayer Mos2. Nano Lett. 2010, 10, 1271-1275. 
56. Gutiérrez, H. R.; Perea-López, N.; Elías, A. L.; Berkdemir, A.; Wang, B.; Lv, R.; López-Urías, F.; Crespi, V. H.; Terrones, H.; Terrones, M., Extraordinary Room-Temperature Photoluminescence in Triangular Ws2 Monolayers. Nano Lett. 2012, 13, 3447-3454.

57. Kobayashi, Y.; Sasaki, S.; Mori, S.; Hibino, H.; Liu, Z.; Watanabe, K.; Taniguchi, T.; Suenaga, K.; Maniwa, Y.; Miyata, Y., Growth and Optical Properties of High-Quality Monolayer Ws2 on Graphite. ACS Nano 2015, 9, 4056-4063.

58. Saakov, V. S.; Drapkin, V. Z.; Krivchenko, A. I.; Rozengart, E. V.; Bogachev, Y. V.; Knyazev, M. N., Derivative Spectrophotometry and Electron Spin Resonance (Esr) Spectroscopy for Ecological and Biological Questions. Springer-Verlag Wien: 2013.

59. Yuan, S.; De Raedt, H.; Katsnelson, M. I., Modeling Electronic Structure and Transport Properties of Graphene with Resonant Scattering Centers. Phys. Rev. B 2010, 82, 115448.

60. Yuan, S.; Roldán, R.; De Raedt, H.; Katsnelson, M. I., Optical Conductivity of Disordered Graphene Beyond the Dirac Cone Approximation. Phys. Rev. B 2011, 84, 195418.

61. Roldán, R.; López-Sancho, M. P.; Guinea, F.; Cappelluti, E.; Silva-Guillén, J. A.; Ordejón, P., Momentum Dependence of Spin-Orbit Interaction Effects in Single-Layer and Multi-Layer Transition Metal Dichalcogenides. 2D Materials 2014, 1, 034003.

62. $\quad$ Perdew, J. P.; Burke, K.; Ernzerhof, M., Generalized Gradient Approximation Made Simple. Phys. Rev. Lett. 1996, 77, 3865-3868.

63. Giannozzi, P.; Baroni, S.; Bonini, N.; Calandra, M.; Car, R.; Cavazzoni, C.; Ceresoli, D.; Chiarotti, G. L.; Cococcioni, M.; Dabo, I., et al., Quantum Espresso: A Modular and Open-Source Software Project for Quantum Simulations of Materials. Journal of Physics-Condensed Matter 2009, $21,395502$.

64. Kresse, G.; Furthmuller, J., Efficient Iterative Schemes for Ab Initio Total-Energy Calculations Using a Plane-Wave Basis Set. Phys. Rev. B 1996, 54, 11169-11186.

65. Kresse, G.; Hafner, J., Ab-Initio Molecular-Dynamics for Open-Shell Transition-Metals. Phys. Rev. B 1993, 48, 13115-13118.

66. Troullier, N.; Martins, J. L., Efficient Pseudopotentials for Plane-Wave Calculations. Phys. Rev. B 1991, 43, 1993-2006.

67. Blochl, P. E., Projector Augmented-Wave Method. Phys. Rev. B 1994, 50, 17953-17979.

68. Kresse, G.; Joubert, D., From Ultrasoft Pseudopotentials to the Projector Augmented-Wave Method. Phys. Rev. B 1999, 59, 1758-1775.

69. Marini, A.; Hogan, C.; Gruening, M.; Varsano, D., Yambo: An Ab Initio Tool for Excited State Calculations. Comput. Phys. Commun. 2009, 180, 1392-1403.

70. Monkhorst, H. J.; Pack, J. D., Special Points for Brillouin-Zone Integrations. Phys. Rev. B 1976, 13, 5188-5192. 